\title{
Three-dimensional flip-flopping flow around a pair of dual-stepped circular cylinders in a
} side-by-side arrangement Yuhao Yan (严雨浩) ${ }^{1}$, Chunning Ji (及春宁) $)^{1,2^{*}}$, Narakorn Srinil ${ }^{3}$

${ }^{1}$ State Key Laboratory of Hydraulic Engineering Simulation and Safety, Tianjin University, Tianjin 300072, China.

${ }^{2}$ Key Laboratory of Earthquake Engineering Simulation and Seismic Resilience of China Earthquake Administration, Tianjin University, Tianjin 300350, China.

${ }^{3}$ School of Engineering, Newcastle University, NE1 7RU, Newcastle upon Tyne, United Kingdom. circular cylinders in a side-by-side arrangement and a proximity interference region are presented. The dual-stepped cylinders consist of two coaxial cylinders with a larger-to-smaller diameter ratio of 2. The Reynolds number and the side-by-side gap ratio, based on the larger (smaller) diameter, is 200

\section{Introduction}

Since the pioneering work of Zdravkovich ${ }^{1}$, several researchers have investigated the proximity

*Corresponding author: cnji@tju.edu.cn 
wake interferences between a pair of side-by-side circular cylinders in cross flow ${ }^{2-4}$. As a gap ratio $\left(g^{*}\right)$ between two side-by-side cylinders varies, different flow interferences appear. For $1-1.2 \leqslant g^{*}$ $\leqslant 5$, the vortex shedding patterns from the paired cylinders become in-phase or anti-phase synchronized. For $0.2 \leqslant g^{*} \leqslant 1.2$, an asymmetric flow regime with a deflected gap flow is observed. An unstable and asymmetric flow pattern has been named as a flip-flopping flow. In a subcritical flow regime with the Reynolds number $(\mathrm{Re})$ of $10^{3} \sim 10^{5}$, the flip-flopping flow features have been reported in several experimental studies ${ }^{5-8}$, exhibiting a gap flow deflecting toward one of the side-by-side cylinders with narrower and wider wake patterns. The cylinder with the narrower wake is subject to a greater drag force coefficient. The biased gap flow intermittently and randomly changes its direction at a low switching frequency. Experimental tests at $g^{*}=0.75$ and $\operatorname{Re}=2-7 \times 10^{3}$ by $\mathrm{Kim}$ and Durbin ${ }^{9}$ showed that such a switching takes place at a frequency being lower, by three orders of magnitude, than the vortex shedding frequency. The flip-flopping flow is typically considered as a dynamical system being divided by two quasi-stable asymmetric states ${ }^{9}$. In turbulence flow simulation at $0.25<g^{*}<0.75$ and $\operatorname{Re}=3000$, an in-phased vortex shedding was reported during the switchover. This phenomenon was reported to be closely associated with the occurrence of large gap vortices by an experiment of $\mathrm{g}^{*}=0.7$ and $150 \leqslant \mathrm{Re} \leqslant 750$. For laminar flow, the two-dimensional simulations of Kang ${ }^{10}$ with $\operatorname{Re}=50 \sim 160$ and $g^{*}=0.4 \sim 1.5$ showed that the gap flow flips over through several vortex shedding periods. In the stability analyses of flow past two side-by-side cylinders in $0.594<g^{*}$ $<0.607$ and $\operatorname{Re} \sim 55$, Mizushima and Ino ${ }^{11}$ revealed a physical origin of the in-phase synchronization regime and the deflected gap flow: i) the flow pattern with a Hopf bifurcation is related to an in-phase synchronization of the vortex shedding, showing an array of paired and aligned vortices behind sideby-side cylinders with the same vortex rotation for each pair but the opposite one for the adjacent pairs; ii) the flow pattern with a pitchfork bifurcation is associated with the deflected gap flow, showing a stationary disturbance extending from the cylinder gap toward downstream. A linear global stability investigation $\left(\mathrm{g}^{*}=0.7, \mathrm{Re} \leqslant 90\right)$ employed by Carini et al. ${ }^{12}$ showed that the flip-flopping state is due to an instability of the in-phase synchronized vortex shedding between the two wakes. They further confirmed that, at a low $50<\operatorname{Re} \leqslant 90$ range, the flip-flop flow is developed though an instability of 
the in-phased vortex shedding. More importantly, an interaction between a perturbation concentrated on the gap flow region (pitchfork bifurcation) and a shedding-like mechanism (Hopf bifurcation) is reported to be at the root of the flip-flop vortex behavior ${ }^{12}$. Recently, analyses based on the dynamic mode decomposition (DMD) $)^{13,14}$ by Liu and Jaiman ${ }^{15,16}$ found DMD modes in the flip-flopping wake region relating to Hopf and pitchfork bifurcations for two side-by-side stationary cylinders.

As cylindrical structures with a spanwise discontinuity in diameter became frequently encountered in several engineering applications, wake patterns of the non-uniform, stepped cylinders consisting of two coaxial circular cylinders with different diameters have recently attracted researchers to study on the three-dimensional vortex structures near the steps. The spanwise discontinuities have been found to have a significant effect on the drag reduction in the cylinder wake ${ }^{17}, 18$. In a three-dimensional simulation case, the vortices are shed from the cylinder in parallel or oblique mode depending on the cylinder aspect ratio $(L / D \text {, length over diameter })^{19}$. A vortex dislocation was observed during the period when vortices in the neighboring cells move out of phase with each other. The vortex shedding becomes closely related to $\operatorname{Re}$ as $L / D$ is less than 7, in terms of the vortex shedding frequency as well as flow characteristics. Three spanwise vortex cells have been classified, including S-cell, N-cell, and L-cell ${ }^{20}$. The S-cell indicates the vortex shedding from the cylinder with a smaller diameter $d$ (referred to as SC hereafter), and with the highest vortex shedding frequency. The L-cell indicates the vortex shedding from the cylinder with a larger diameter $D$ (referred to as LC hereafter), and with a vortex shedding frequency lower than that of the S-cell. The N-cell indicates the vortex shedding from the step on the LC side, with the lowest vortex shedding frequency. Direct and indirect connection modes have been found in the experiment of Lewis and Gharib ${ }^{21}$ for laminar flow with $\operatorname{Re}=35 \sim 200$. The direct mode occurs when $D / d<1.25$, with two distinct vortex shedding frequencies corresponding to SC and LC, respectively. The indirect mode occurs when $D / d>1.55$, with a third frequency (lower than the two aforementioned frequencies) being discovered in the near step wake being named the modulated zone. This zone was also reported in the turbulent flow behavior of single-step cylinders ${ }^{22}$, indicating a similarity between laminar and turbulent flows ${ }^{21}$. Further, a half-loop connection between the two counter-rotating spanwise vortex tubes shed from SC were reported in the experimental study of Lewis and Gharib ${ }^{21}$, and subsequently in the computational study of Vallès et al. ${ }^{23}$. Numerical simulations of Morton and Yarusevych ${ }^{24}$ with $D / d=2$ at $\mathrm{Re}=150$ and 300 showed that a connective 
region between $\mathrm{S}$-cell and $\mathrm{N}$-cell is relatively narrow and stable. A vortex dislocation, appearing as a half-loop connection, occurs between two S-cell vortices. However, a connection region between Ncell and L-cell is wider, with N-cell varying periodically during the vortex dislocation. A further investigation into the vortex dislocation together with antisymmetric vortex interactions was reported in ${ }^{25}$ under the same configuration. Recently, Tian et al. ${ }^{26}$ numerically studied the vortex dislocation of a stepped cylinder with $D / d=2$ and 2.4 at $\operatorname{Re}=150$. They revealed that a phase difference between $\mathrm{N}$-cell and L-cell is accumulated due to the distinct vortex shedding frequencies and convective velocities of these two cells.

"Two-sided" vortex dislocations were found in the wake of a uniform cylinder with a small ring disturbance, generating a dual-stepped cylinder configuration ${ }^{27}$, where a forced vortex dislocation can be obtained for $\operatorname{Re}_{\mathrm{D}} \leqslant 200,1.1 \leqslant D / d \leqslant 2$ and $L / d=0.5$. Later, the effect of aspect ratio of the larger cylinder on the vortex shedding at $\operatorname{Re}_{\mathrm{D}}=1050$ and $D / d=2$ was studied by Morton and Yarusevych $^{28}$. Four distinct vortex shedding patterns were identified for $0.2<L / D<17$, based on the vortex dynamics in the LC wake. A more detailed classification of variations in the LC wake topology for $2000 \leqslant \operatorname{Re}_{\mathrm{D}} \leqslant 5500,1.33 \leqslant D / d \leqslant 2$ and $0.5 \leqslant L / d \leqslant 5$ was reported in ${ }^{29}$. Recently, a detailed map of six distinct flow regimes of flow around a dual-stepped cylinder for $\mathrm{Re}_{\mathrm{D}}=2100$ was reported in $\operatorname{Ref}^{30}$, for the range of $0.2 \leqslant L / d \leqslant 5$ and $1.33 \leqslant D / d \leqslant 4$. In laminar flow regime, two new vortex interactions (double-half-loop and three-half-loop connections) were found in the wake of a dual-stepped cylinder at $\mathrm{Re}=200^{31}$. Three distinct vortex shedding modes were reported: the N$\mathrm{S}$ mode $(D / d=2)$, the transition mode $(D / d=1.43)$, and the L-S mode $(D / d=1.19)$. Moreover, in the wake of a dual-stepped circular cylinder undergoing vortex-induced vibration, a new "X-shaped" connection was discovered by Ji et al. ${ }^{32}$.

From the above literature review, the flip-flopping flow of two side-by-side uniform cylinders has been extensively investigated. Nevertheless, the more complex vortex shedding modes, wake bifurcation features and hydrodynamic forces associated with the flip-flopping flow mechanism of two side-by-side dual stepped cylinders with a spanwise geometric discontinuity have not been studied. In this paper, we investigate the three-dimensional vortex dynamics of a pair of dual-stepped circular cylinders in a side-by-side configuration through the direct numerical simulation. To the best of the 
authors' knowledge, this fluid dynamic problem is herein studied for the first time. The objectives of the present study are three-fold: (i) to investigate the effect of dual steps on the flip-flopping flow characteristics, (ii) to ascertain the influence of the deflected gap flow on the vortex dynamics, and (iii) to identify the origin of the flip-flopping flow in the wake of dual-stepped cylinders in a side-by-side configuration.

At the present time, our computation capabilities for 3-D direct numerical simulations are limited to a low Re range for laminar flow. Hence, we have selected the standard Re values for both smaller $\left(\operatorname{Re}_{d}=100\right)$ and larger $\left(\operatorname{Re}_{D}=200\right)$ diameters to be within the laminar flow regime. For the proximity interference wake regime, a gap-to-diameter ratio for a pair of side-by-side cylinders has been identified to be about 3 or less ${ }^{2}$. Further, a flip-flopping flow behavior has previously been observed for a pair of side-by-side uniform cylinders with a gap ratio of 1.2 or less $^{2}$. By keeping these relatively narrow ranges in mind, we assign a diameter ratio $D / d=2\left(\operatorname{Re}_{D}=200, \operatorname{Re}_{d}=100\right)$ and the associated gap ratios $\left(g^{*}{ }_{D}=1, g^{*}=3\right)$ such that a 3-D flip-flopping flow characteristics would be expected for the larger cylinder (i.e. $g^{*}<1.2$ ) within the proximity interference regime. Then, the influence of the deflected gap flow on the vortex dynamics with various wake patterns can be investigated, with an identification of the origin of the flip-flopping flow. These will be thoroughly discussed in Section 3 with the above-selected parameters. To demonstrate the combined effects of Re, gap ratio and diameter ratio, additional discussion and simulation results are supplemented in the Appendix for the dualstepped side-by-side cylinders with a smaller $D / d=1.33$. In this case, the smaller diameter is unchanged whereas the larger one is reduced such that $\operatorname{Re}_{D}$ becomes 133.3 , while $\operatorname{Re}_{d}=100$. Thus, the larger cylinder gap ratio becomes $g{ }^{*}=2$, while $g{ }_{d}=3$ remains unchanged. Results in the Appendix reveal a simpler vortex shedding pattern with stable and periodic anti-phase synchronized wake for both larger and smaller cylinders without showing a flip-flopping behavior. Therefore, these results justify the considered flow geometry and configuration, with the aim of capturing the 3D flip-flopping flow within the proximity interference regime in laminar flow, apart from revealing the combined influence of Re, diameter ratio and gap ratio when varying the larger diameter.

The rest of the paper is organized as follows. Section 2 presents the numerical methodology and computational setup. Three-dimensional flow evolutions and vortex structures are studied in Sections 3.1 and 3.2, respectively. Two- and three-dimensional DMD modes of the wake and their connections 
141 with flow features are discussed in Section 3.3. The paper ends with the main findings and key conclusions in Section 4.

\section{Numerical methodology}

\subsection{Governing equations}

The three-dimensional flow past a pair of dual-stepped circular cylinders is governed by the incompressible continuity and Navier-Stokes equations,

$$
\begin{gathered}
\nabla \cdot \mathbf{u}=0 \\
\frac{\partial \mathbf{u}}{\partial t}+(\mathbf{u} \cdot \nabla) \mathbf{u}=-\frac{1}{\rho} \nabla p+v \nabla^{2} \mathbf{u}
\end{gathered}
$$

where $u$ is the velocity vector, $t$ the time, $\rho$ the fluid density, $p$ the pressure, and $v$ the kinematic viscosity.

These equations are numerically solved by applying the open-source framework Nektar $++{ }^{33-35}$ using the spectral/hp element method ${ }^{36}$. The fourth-order polynomials are adopted, achieving an improved numerical stability. We have found that higher-order polynomials may break down some simulations. A high-order splitting scheme ${ }^{37}$ is employed for the time integration by using an approach proposed in Guermond and Shen ${ }^{38}$, see https://www.nektar.info/ for more details.

\subsection{Dynamic Mode Decomposition (DMD)}

A Dynamic Mode Decomposition (DMD) method ${ }^{14,39}$ is employed to capture intrinsic features of the flip-flopping flow. In so doing, two series of the flow snapshots within a time interval $\Delta t$ are expressed as:

$$
\begin{aligned}
& X=\left[x_{1}, x_{2}, \cdots, x_{m-1}\right] \\
& X^{\prime}=\left[x_{2}, x_{3}, \cdots, x_{m}\right]
\end{aligned}
$$

A leading decomposition of the best-fit linear operator $A$ satisfying:

$$
X^{\prime} \approx A X
$$

is then performed. An eigenvector $\phi_{k}(k=1,2, \ldots, r$, where $r$ is the rank of the reduced SVD approximation to $X)$ of $A$ represents each DMD mode. The corresponding eigenvalue $\mu_{k}(k=1,2, \ldots$, $r$ ) contains key information with the real part $\mu_{r}$ representing the growth/decaying rate of the mode and the imagine part $\mu_{i}$ indicating the rational frequency. 
To perform a low-rank truncation of the data, the SVD reduction is performed:

$$
X \approx U \Sigma V^{*}
$$

where $U \in \mathbb{C}^{n \times r}$ is the left singular vector with orthonormal columns, also known as POD modes, $\Sigma \in$ $\mathbb{C}^{r \times r}$ is a diagonal matrix composed of singular values, $V \in \mathbb{C}^{m \times r}$ is the right singular vector, and the superscript $*$ represents the conjugate transpose. Here, $n$ denotes the number of data points in each snapshot.

The matrix A in Eq. (12) is obtained by using the pseudo-inverse of $X$ :

$$
A=X^{\prime} V \Sigma^{-1} U^{*}
$$

However, it is more effective to compute $\widetilde{A}$, a $r \times r$ projection of the full matrix $A$ onto the POD modes, which defines a low-dimensional linear model of the dynamic system as $\tilde{x}_{k+1}=\widetilde{A} \tilde{x}_{k}$, with $x_{k}=U \tilde{x}_{k}$. By computing the eigen-decomposition of $\widetilde{A}$ :

$$
\widetilde{A} W=W \Lambda
$$

where each column in $W$ represents an eigenvector with its eigenvalue $\mu_{k}$ being the corresponding diagonal element in $\Lambda$. Now, eigenvalues of $A$ are given by diagonal elements in $\Lambda$, and the eigenvectors of $A$ are given by columns in $\Phi$ :

$$
\Phi=X^{\prime} V \Sigma^{-1} W
$$

The DMD frequency $f_{D M D}$ is then calculated as:

$$
f_{D M D}=\frac{\ln \left(\mu_{i}\right)}{2 \pi \Delta t}
$$

\subsection{Computational setup}

Schematics of a pair of identical dual-stepped circular cylinders in a side-by-side configuration and the simulation domain are illustrated in Figure 1. The cylinders have a larger-to-smaller diameter ratio $D / d=2$, with a side-by-side gap ratio $g^{*}{ }_{D}=1(\mathrm{gap} / D)$ for $\mathrm{LC}$ and $g^{*}{ }_{d}=3(\mathrm{gap} / d)$ for SC. The overall aspect ratio $L / D=10$, where $L$ represents the total cylinder length. LC has a spanwise length of $L_{D}=5 D$ while each SC has a spanwise length of $L_{d}=2.5 D$ for each top and bottom part, see Figure 1(a). For a given freestream velocity $U_{\infty}$, the associated Reynolds number is $\operatorname{Re}_{D}=U_{\infty} D / v=200$ for LC, yielding $\operatorname{Re}_{d}=U_{\infty} d / v=100$ for SC. In Figure 1(b), the computational domain is set as [-10D, $15 D] \times[-10 D, 10 D] \times[0,10 D]$ in $x, y$ and $z$ direction, respectively, with the origin locating at the mid- 
point between the two cylinders at the bottom surface $(z=0)$. The inflow boundary with the Dirichlet boundary conditions $u=U_{\infty}, v=w=0$ is $10 D$ upstream the center of the cylinders, while the outflow boundary with the Neumann boundary conditions $\partial u / \partial x=\partial v / \partial x=\partial w / \partial x=0$ is $15 D$ downstream. The upper and lower surfaces are set as periodic boundaries, while the front and back surfaces are set as

where $C_{D, L}\left(C_{D, S}\right)$ and $C_{L, L}\left(C_{L, S}\right)$ are the drag and lift coefficients of larger (smaller) cylinder, $F_{D, L}\left(F_{D, S}\right)$ and $F_{L, L}\left(F_{L, S}\right)$ are the drag and lift forces on the larger (smaller) cylinder, respectively, $F_{D, \text { step }}$ and $F_{L, s t e p}$ are the total drag and lift forces on the two step surfaces at $Z / D=2.5$ and 7.5 , respectively.

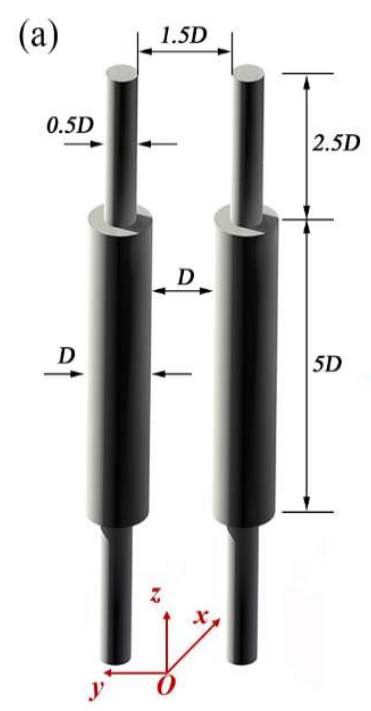

$$
\begin{gathered}
C_{D, L}=\frac{2\left(F_{D, L}+F_{D, \text { step }}\right)}{\rho U_{\infty}^{2} D L_{D}}, C_{D, S}=\frac{2 F_{D, S}}{\rho U_{\infty}^{2} d L_{d}} \\
C_{L, L}=\frac{2\left(F_{L, L}+F_{L, \text { step }}\right)}{\rho U_{\infty}^{2} D L_{D}}, C_{L, S}=\frac{2 F_{L, S}}{\rho U_{\infty}^{2} d L_{d}}
\end{gathered}
$$

Figure 1. Computational setup: (a) side-by-side, dual-stepped cylinders, (b) simulation domain.

A structured mesh is generated by the three-dimensional finite element mesh generator Gmsh, as shown in Figure 2. To guarantee accuracy of simulation results, the mesh is refined near the cylinders in a region of $2 D$ in the $x$ direction and $4 D$ in the $y$ direction, see Figure 2(b). To capture the vortex 
212 structures near the steps, a smaller spanwise grid spacing is adopted near the steps, see Figure 2(c). A 213 grid dependency test is first carried out to make sure that the numerically obtained results are grid 214 independent. Three mesh densities are adopted for the three-dimensional flow past two side-by-side 215 dual-stepped cylinders with a diameter ratio $D / d=1.33, \operatorname{Re}_{d}=100\left(\operatorname{Re}_{D}=133.33\right)$ and $g *_{d}=3\left(g^{*}{ }_{D}=\right.$ 216 2). As shown in Figure A1 in the Appendix, the wake shows an anti-phase synchronization (AS) flow 217 pattern, and the force statistics of the two cylinders become identical if the averaging time is 218 sufficiently long. From Table 1, it is noticed that results for Mesh 2 converge. Hence, Mesh 2 is applied 219 in the simulation of flow past two side-by-side uniform cylinders at $\operatorname{Re}=100$ and $g *=0.7$ for validation. 220 Table 2 compares the present results with those in Kang ${ }^{10}$,Wang et al. ${ }^{40}$, Lee et al. ${ }^{41}$, Chen et al. ${ }^{42}$. As 221 shown in Table 2, a reasonable agreement is achieved in terms of hydrodynamic forces. The mean drag 222 coefficient $C_{D, \text { mean }}$ and the root-mean-square (r.m.s.) lift coefficient $C_{L, r m s}$ appear to be slightly scattered 223 owing to the different computational widths adopted in the referenced studies. In the following 224 simulations, Mesh 2 is employed.

Table 1. Grid convergence study of three-dimensional flow past two side-by-side dual-step cylinders with diameter ratio $D / d=1.33, \operatorname{Re}_{d}=100\left(\operatorname{Re}_{D}=133.33\right)$ and $g^{*}{ }_{d}=3\left(g^{*}{ }_{D}=2\right) . C_{D}$ and $C_{L}$ are the drag and lift coefficients of SC, respectively. Subscripts mean and $r m s$ represent the time-averaged and rootmean-square values, respectively.

\begin{tabular}{ccccc}
\hline \hline Mesh & Elements & $C_{D, \text { mean }}$ & $C_{L, \text { rms }}$ & $S t$ \\
\hline 1 & 338720 & 2.2196 & 1.4700 & 0.1771 \\
2 & 436160 & 2.2220 & 1.4815 & 0.1842 \\
3 & 468000 & 2.2198 & 1.4819 & 0.1849 \\
\hline \hline
\end{tabular}


(a)

(b)

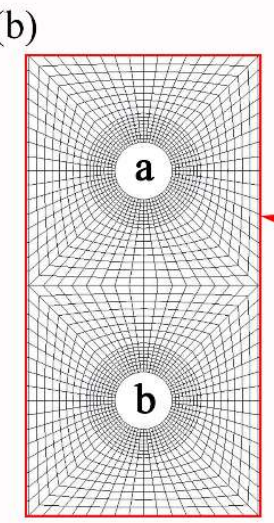

(c)

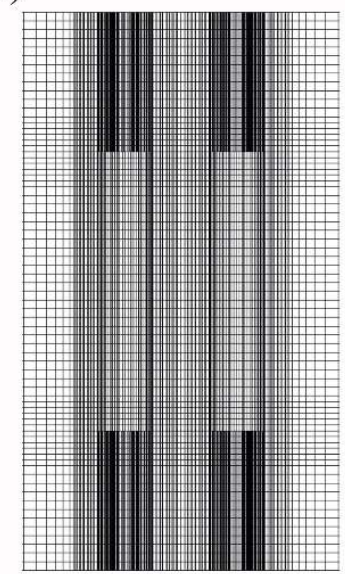

233 Figure 2. Computational mesh on (a, b) $x-y$ plane and a close-up view, and (c) $x-z$ plane.

Table 2. Comparison between the present results and those in the literature for flow past a pair of uniform cylinders in side-by-side arrangement at $\mathrm{Re}=100$ and $g^{*}=0.7$.

\begin{tabular}{ccc}
\hline \hline & $C_{D, \text { mean }}$ & $C_{L, \text { rms }}$ \\
\hline Kang $^{10}$ & 1.3892 & 0.3640 \\
Wang et al. $^{40}$ & 1.6268 & 0.4198 \\
Lee et al. $^{41}$ & 1.4003 & 0.3490 \\
Chen et al. $^{42}$ & 1.3970 & 0.3571 \\
Present (Mesh 2) & 1.5834 & 0.3851 \\
\hline \hline
\end{tabular}

\section{Results and Discussion}

To visualize the instantaneous three-dimensional vortex structures, the iso-surface of the Qcriterion at $\mathrm{Q}=0.2$ is presented in Figure 3(a,b), corresponding to the time instants when the gap flow deflects toward the upper cylinder [a] and the lower cylinder [b], respectively. The iso-surface is colored by the z-vorticity to differentiate the clockwise (gray) and anti-clockwise (blue) vortex tubes. The vortex structures can be divided into two cells: N-cell downstream of LC and S-cell downstream of SC. As expected, a flip-flopping flow pattern is found in the LC wake with a deflected gap flow (see

244 Figures $3 \mathrm{c}$ and 3e), leading to a narrow-wide wake pattern which is analogous to the observations 
245 reported in the literature ${ }^{10,12,16}$. Specifically, one can recognize a relatively narrower wake behind 246 cylinder [b] and a wider one behind cylinder [a] in Figure 3(c), indicating a downward biased gap flow. 247 In Figure 3(e), the gap flow flips over, leading to a wider wake behind cylinder [b] and a narrower 248 wake behind cylinder [a]. This depicts an alternately deflected gap flow. It is worth noting that a flip249 flopping-like pattern also develops in the SC wake. This is supposed to be an anti-phase 250 synchronization (AS) regime, featured by two rows of parallel vortex streets in the anti-phase fashion, 251 for two side-by-side uniform cylinders at $g^{*}{ }_{d}=3$ and $\operatorname{Re}_{d}=100{ }^{10,16}$. However, compared to the LC 252 flip-flopping flow, a clear difference is observed for which the gap flow between the smaller cylinders 253 shows a negligible deflection and, thus, the narrow-wide wake pattern disappears. Instead, a short-long 254 wake pattern, analogous to the flip-flopping wake pattern of two free-vibrating side-by-side cylinders $255^{43,44}$, is observed.

256 It should be noted that the wake pattern of the dual-stepped cylinders in side-by-side arrangements 257 are highly sensitive to the diameter ratio, the Reynolds number and the gap ratio. To demonstrate this 258 aspect, additional simulations with a smaller diameter ratio of $D / d=1.33$ are performed whose results are presented in the Appendix. In this scenario, we keep the smaller diameter unchanged but reduce the larger one such that the associated $\mathrm{Re}_{D}$ becomes smaller and equal to 133.3. Accordingly, a larger gap ratio $g^{*}{ }_{D}=2$ is considered for the larger diameter. For smaller diameter, $\operatorname{Re}_{d}=100$ and $g^{*}{ }_{d}=3$ are unchanged. This smaller $D / d$ configuration triggers a simpler vortex shedding pattern with a stable and periodic anti-phase synchronized wake for both LC and SC, without exhibiting a flip-flopping flow feature. These results suggest the combined influence of Re, diameter ratio and gap ratio when varying the larger diameter. 
(a)

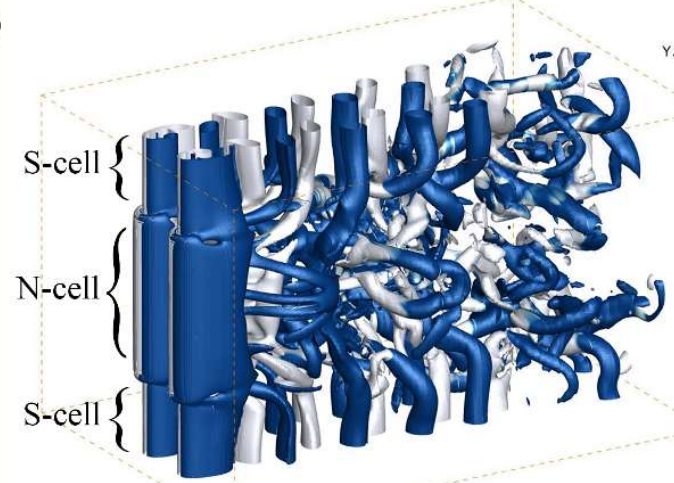

(c)

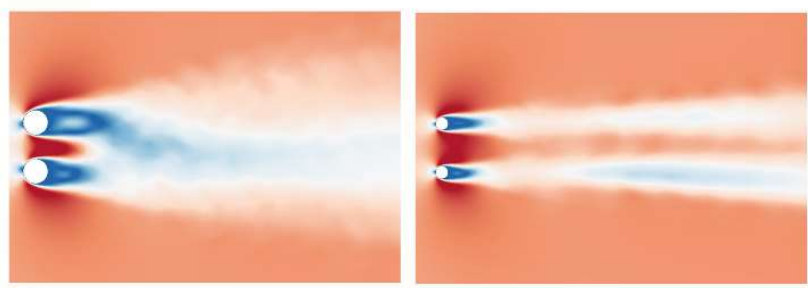

(b)

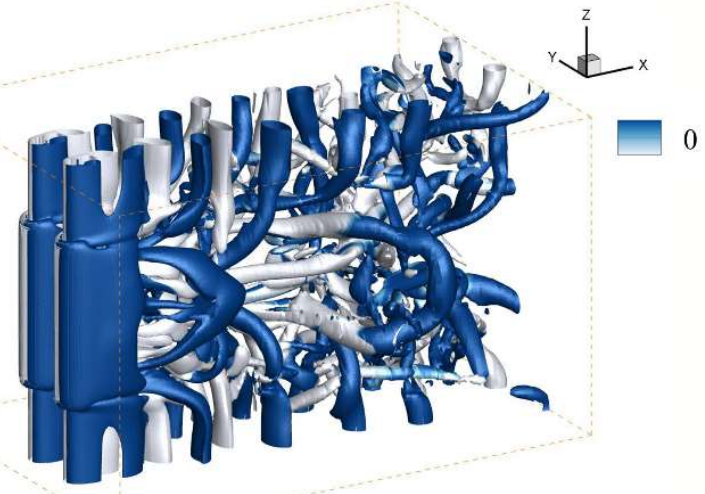

(e)

(f)
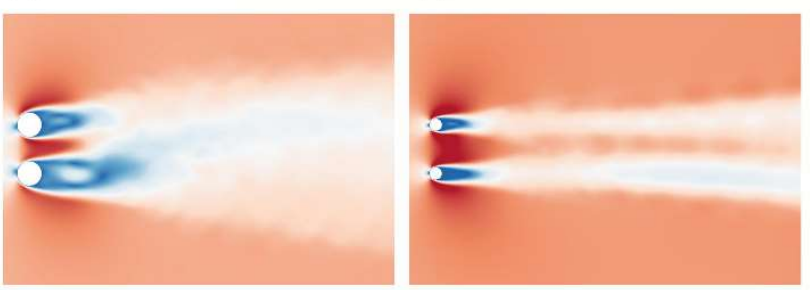

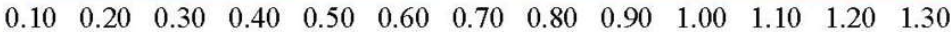

Figure 3. Instantaneous vortex structures, visualized by the iso-surface of the $\mathrm{Q}$-criterion at $\mathrm{Q}=0.2$, downstream of side-by-side dual-stepped cylinders ( $a$ and $b$ ), and the time-averaged streamwise velocity fields (c - f). Subplots (c) and (e) depict the flow field at $z / D=5.5$ while subplots (d) and (f) depict the flow field at $z / D=9.5$.

\subsection{Hydrodynamic force coefficients}

Overall, the time evolutions of $C_{D}$ and $C_{L}$ for both cylinders manifest the similar flip-flopping characteristics, as shown in Figure 4 . The $C_{D}$ value of the cylinder with a narrower wake is relatively greater. However, this feature flips over irregularly and intermittently for which a switching period is about one order of the magnitude longer than the vortex shedding period. The amplitude envelope of $C_{L}$ also shows wavy variations, following the $C_{D}$ pattern and amplitude trend. A similar flip-flopping flow feature for a pair of side-by-side cylinders with a uniform diameter has been reported in Kang ${ }^{10}$.

By further inspecting $C_{D}$ and $C_{L}$ of LC versus $\mathrm{SC}$, some qualitative differences can be noticed. Although both LC and SC have the same low-frequency and in-phase variations in $C_{D}$ and $C_{L}$ originating from the flip-flopping flow, the high-frequency variations in $C_{D}$ and $C_{L}$, caused by the vortex shedding, have a greater frequency value for SC than that for LC. This indicates how SC has a 
faster vortex shedding leading to the vortex dislocation, as further discussed in Section 3.2. Moreover, the two alternate and irregular flow regimes A and B, indicated with the orders ( 1 to 3 ) in Figure 4, are identified, with the former regime having a lower flip-flopping frequency. Both $C_{D}$ and $C_{L}$ amplitudes in regime $\mathrm{B}$ are greater than those in regime $\mathrm{A}$.
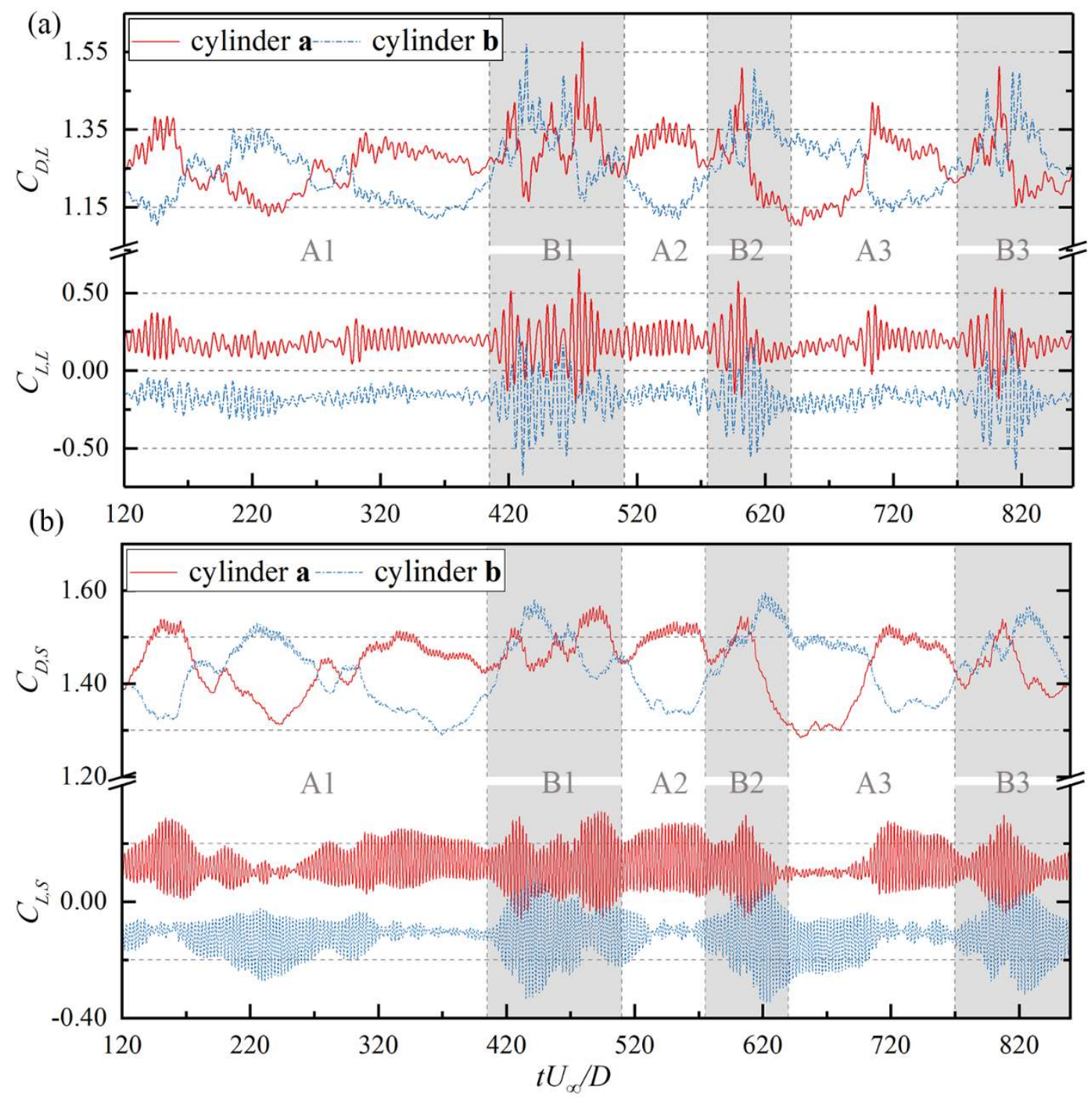

Figure 4. Time evolutions of $\operatorname{drag}\left(C_{D}\right)$ and lift $\left(C_{L}\right)$ force coefficients for dual-stepped cylinders in a side-by-side arrangement: (a) LC, (b) SC, with two distinct flow regimes A and B. Cylinders [a] and [b] are the upper and lower cylinders, respectively.

The velocity fluctuations of the shear layers are highly correlated with the flip-flopping switching and vortex shedding periods. In this study, the instantaneous streamwise velocity is measured along the two spanwise lines at $x / D=5$, and $y / D=0.5$ and 1.5 , which are downstream of cylinder [a], and 
294 aligned with the cylinder bottom and top, respectively. Figure 5 shows the space-time variations of the streamwise velocity $u_{\text {in }}$ (for the gap-side shear layer) and $u_{o u t}$ (for the freestream-side shear layer) within three consecutive periods of $240<t U_{\infty} / D<360$ (A1), $510<t U_{\infty} / D<570$ (A2), and $640<t U_{\infty} / D$ $<770$ (A3). For A1 and A3, the vortex shedding of LC reveals two distinct stages, with the first stage exhibiting a lower $u$ downstream of LC. This is attributed to the gap flow deflection. As the gap flow is downward biased in the first stage, the measuring lines are fully submerged in the recirculation region of LC due to the wider wake of cylinder [a]. This entails a lower streamwise velocity. On the other hand, when the gap flow is upward deflected in the second stage, the measuring lines are outside the recirculation region, resulting in a higher streamwise velocity. This trend is less significant for the freestream-side shear layer than for the gap-side shear layer. Different from A1 and A3, a flip-flopping switching does not occur in A2 (see Figure 4). As a result, the velocity distribution behind cylinder [a] with a narrower wake shows a consistent pattern within this period. A similarity in the space-time distribution of $u$ can be found between $\mathrm{A} 2$ and the second stage in A1 and A3.

Besides the prevailing differences related to the flip-flopping flow, the space-time distribution of $u$ also reveals some differences in the vortex shedding of SC. It is found that a spacing between the two velocity stripes in the first stage is wider than that in the second stage, i.e. $S_{w}>S_{n}$, as shown in Figures 5(a) and 5(c). This indicates that the vortex shedding frequency of SC varies with the gap flow switching. SC sheds vortices faster when it has a narrower wake. This is consistent with the findings of Chen et al. ${ }^{44}$ Chen et al. ${ }^{45}$ who reported that the cylinder with a narrower wake has a higher vortex shedding frequency. Different from regime A, the space-time distribution of $u$ in $\mathrm{B} 1$ to $\mathrm{B} 3$ shows no distinct stages, as shown in Figure 6. The $u$ variations are irregular in the wake of both SC and LC, implying the complicated and fast changing vortex structures in regime B. 


$$
\text { (c) }
$$

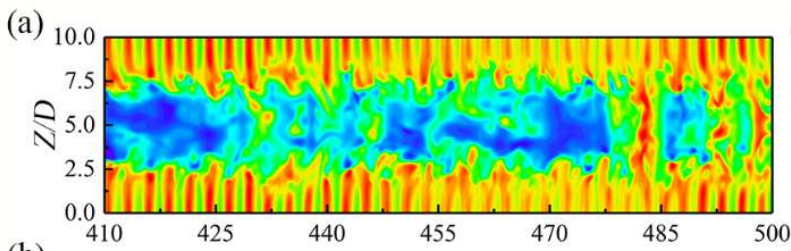

(b)
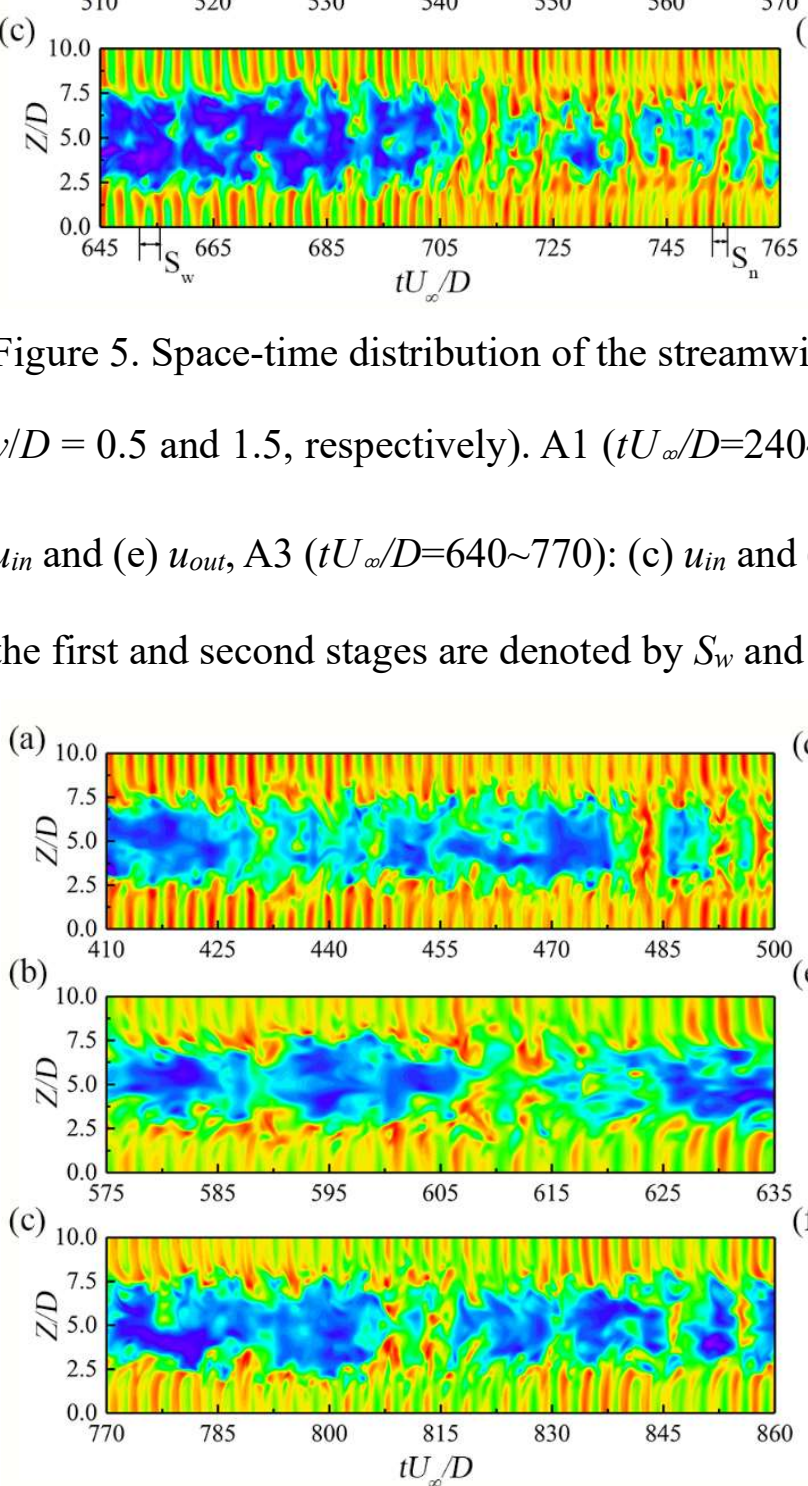

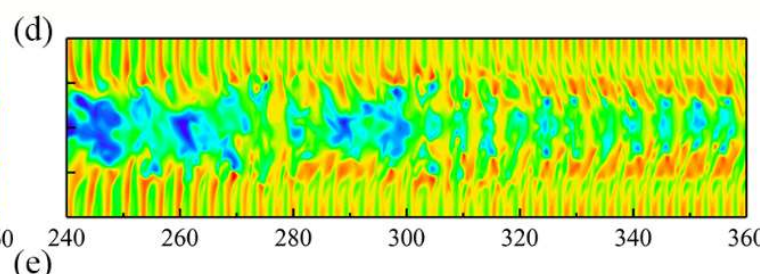

(e)

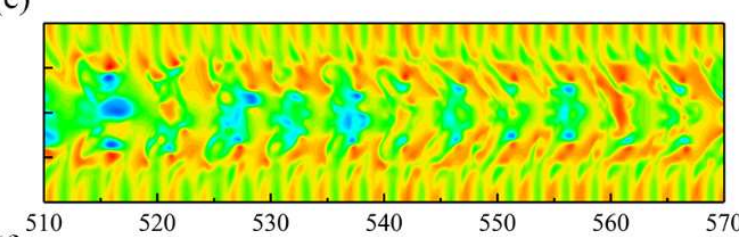

(f)

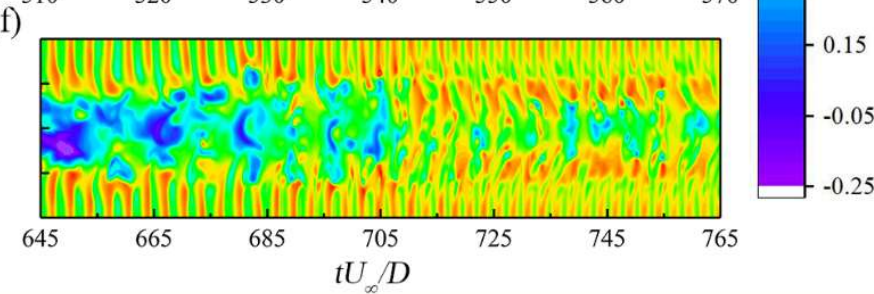

Figure 5. Space-time distribution of the streamwise velocity measured at two spanwise lines $(x / D=5$, $y / D=0.5$ and 1.5, respectively). A1 ( $\left.t U_{\infty} / D=240 \sim 360\right)$ : (a) $u_{i n}$ and (d) $u_{o u t}, \mathrm{~A} 2\left(t U_{\infty} / D=510 \sim 570\right)$ : (b) $u_{\text {in }}$ and (e) $u_{\text {out }}, \mathrm{A} 3\left(t U_{\infty} / D=640 \sim 770\right)$ : (c) $u_{\text {in }}$ and (f) $u_{o u t}$. A spacing between the two velocity stripes in the first and second stages are denoted by $S_{w}$ and $S_{n}$, respectively.

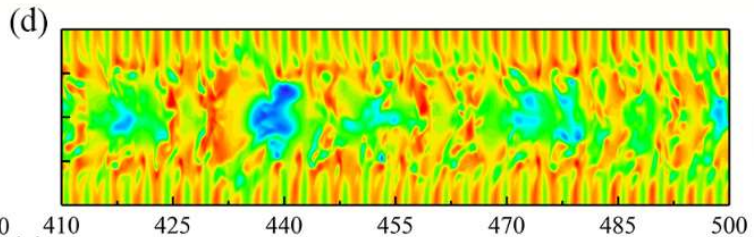

(e)

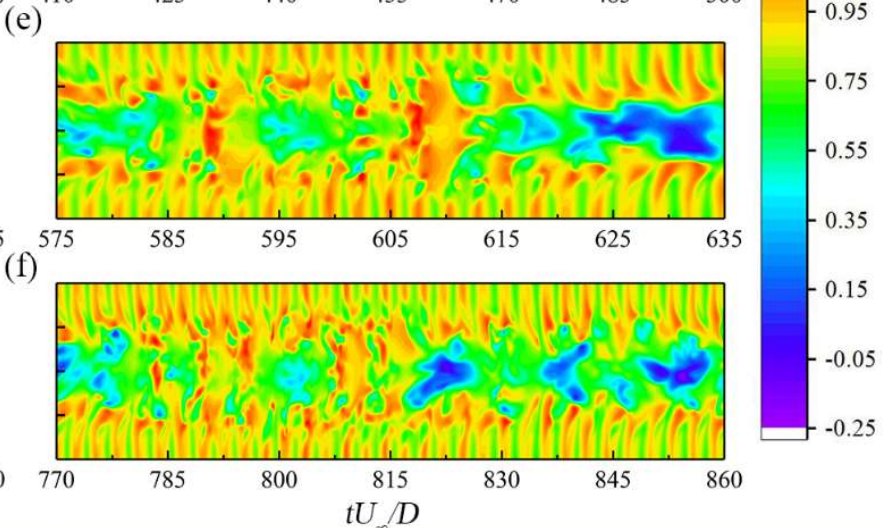

Figure 6. Space-time distribution of the streamwise velocity measured at two spanwise lines $(x / D=5$, $y / D=0.5$ and 1.5 , respectively). B1 $\left(t U_{\infty} / D=410 \sim 500\right):$ (a) $u_{\text {in }}$ and (d) $u_{\text {out }}, \mathrm{B} 2\left(t U_{\infty} / D=575 \sim 635\right):$ (b) 
Next, the frequency characteristics of the wake flow are investigated by applying the Fast Fourier Transform (FFT) of the instantaneous streamwise $(u)$ and transverse $(v)$ velocities probed at $x / D=5$ and $y / D=1.5$. Two periods $\mathrm{A} 1\left(t U_{\infty} / D=120.7 \sim 345.7\right)$ and $\mathrm{B} 1\left(t U_{\infty} / D=415.7 \sim 507.7\right)$ are selected, assuring that at least one complete flip-flopping flow cycle is captured (see Figure 4). Figure 7 shows the power spectral density (PSD) $\underline{E}_{u u}$ and $E_{v v}$ (see $\operatorname{Ref}^{46}$ for definitions) normalized by the total power density. Clearly, the spectra in these two flow regimes show some differences. Firstly, the flip-flopping frequency is recognized as $f_{F F}=0.018$ in $\mathrm{A} 1$ but $f_{F F}=0.054$ in $\mathrm{B} 1$ : this provides a solid evidence of the aforementioned finding that the flip-flopping behavior takes place at a higher frequency in regime B than in regime A. Secondly, the vortex shedding frequencies are $f_{S}=0.356$ and 0.370 in A1 and B1, respectively, for S-cell, and $f_{N}=0.129$ and 0.152 , respectively, for $\mathrm{N}$-cell. This indicates that not only the frequency of the flip-flopping switching, but also the vortex shedding frequency is slightly higher in regime B. Finally, the ratios between the dominant frequencies related to the vortex shedding of Ncell and S-cell are non-integer in both flow regimes, i.e. $f_{S} / f_{N} \approx 2.76$ in $\mathrm{A} 1$ and 2.43 in $\mathrm{B} 1$. This implies that the vortex connections between $\mathrm{N}$-cell and S-cell are aperiodic and irregular. The non-integer frequency ratio was also found in Ji et al. ${ }^{31,32}$.

(a)

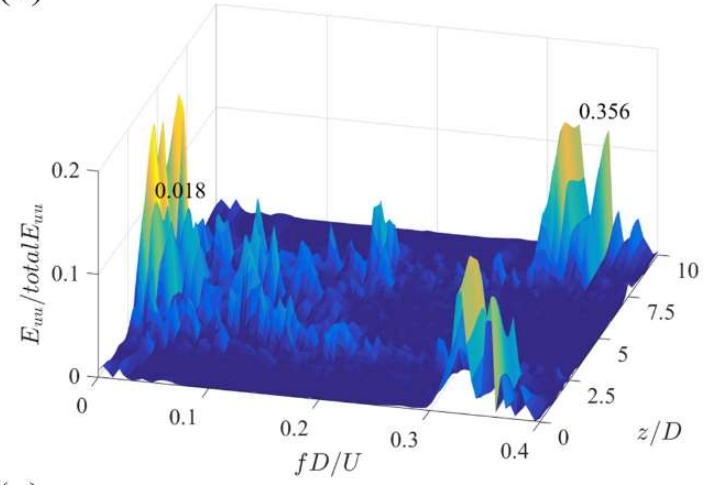

(c)

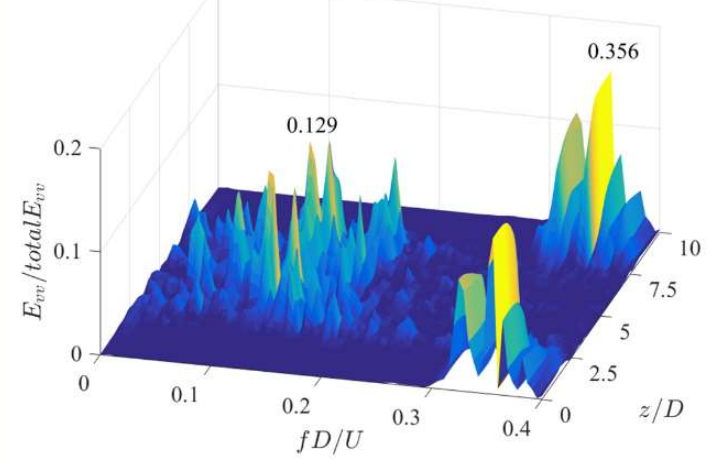

(b)

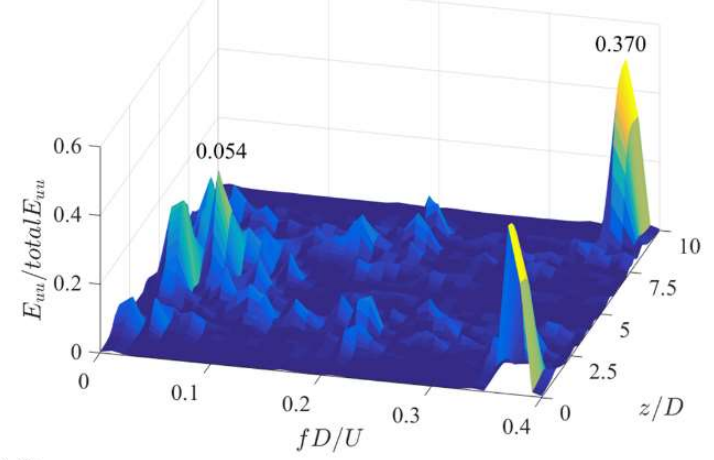

(d)

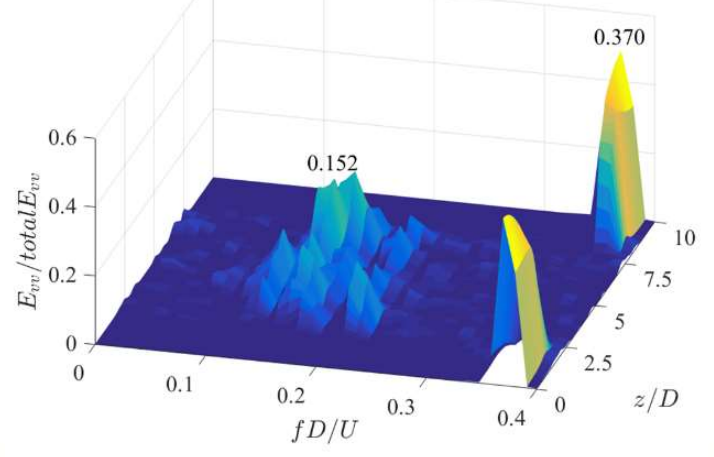


341 Figure 7. PSD spectra of the normalized energy density in the streamwise and transverse directions, 342 respectively, in $\mathrm{A} 1\left(t U_{\infty} / D=120.7 \sim 345.7\right)$ and $\mathrm{B} 1\left(t U_{\infty} / D=415.7 \sim 507.7\right)$. (a) and (c) show the spectra 343 of normalized $E_{u u}$ and $E_{v v}$ in A1, respectively, (b) and (d) show the spectra of normalized $E_{u u}$ and $E_{v v}$ 344 in B1, respectively.

345 A phase lag between the flip-flopping-related drag components of side-by-side cylinders is further 346 investigated, together with that of the lift amplitude envelope. Based on FFT results, the flip-flopping 347 frequency is $f_{F F} \sim 0.01$, while that of the vortex shedding is $f_{S} \sim 0.3$ and $f_{N} \sim 0.1$ for SC and LC, 348 respectively. Thus, a filter band [0.0001, 0.08] is adopted to extract the flip-flopping drag frequency 349 by excluding the vortex shedding interference. As shown in Figure 8(a), the phase lag of the flip350 flopping components of $C_{D}$ is close to $180^{\circ}$ in regime $\mathrm{A}$, but it shows large fluctuations in regime $\mathrm{B}$, 351 for both SC and LC. On the other hand, for the amplitude envelope of $C_{L}$, some differences between $352 \mathrm{SC}$ and LC behaviors emerge. For SC, variations of the $C_{L}$ amplitude envelope are predominantly anti353 phase in regime A but out-of-phase in regime B, showing a good similarity with the flip-flopping drag. 354 However, for LC, the phase lag is close to zero in regime A, in spite of existing large fluctuations. 

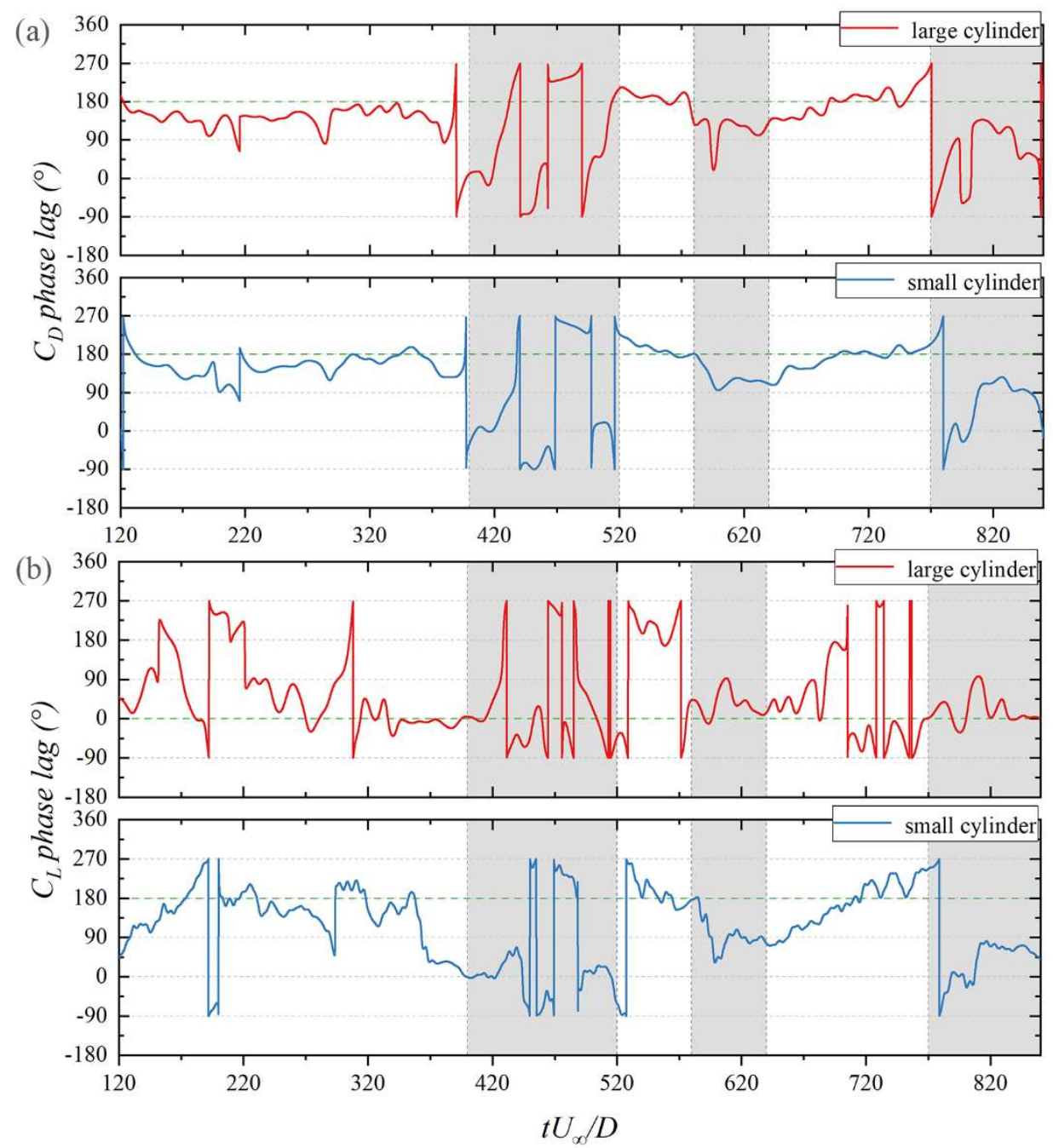

\subsection{Vortex structures}

To visualize the complex three-dimensional vortex structures in the wake of side-by-side, dualstepped cylinders exhibiting the flip-flopping gap flow, the iso-surface of the Q-criterion at $\mathrm{Q}=0.2$ are presented in Figures 9 and 10 together with the associated simplified sketches in Figure 11. Figure 9 displays the vortex structures downstream of cylinder [a] with a narrower wake, by discarding the wake vortices of cylinder [b] for a clearer illustration. The iso-surface plots in Figure 9 exhibit the streamwise-oriented junction, the edge and center vortices, and also the spanwise-oriented S-cell and $\mathrm{N}$-cell vortices. The half-loop vortex connection ${ }^{12,13}$ between the two counter-rotating S-cell vortices and the direct vortex connection between the co-rotating S-cell and N-cell vortices are observed. These vortices in the narrower wake appear to be similar to those of a single dual-stepped cylinder in view of the half-loop and direct vortex connections taking place in a sequence. It should also be noticed that 
368 the gap-side N-cell vortices are quickly damped out and disappear downstream due to a strong vortex 369 interaction with their counterparts of cylinder [b]. However, the gap-side S-cell vortices are relatively 370 affected, owing to the larger transverse spacing between smaller cylinders.

371 Due to a lower streamwise convective velocity downstream of LC than that downstream of SC, 372 there exists a reconnection between N-cell and S-cell vortices. Figure 9 shows the evolution of vortex 373 structures in a complete vortex shedding period with one and two vortex pairs being shed from LC and $374 \mathrm{SC}$, respectively. At $t U_{\infty} / D=425.7$ when $\mathrm{N} 1$ is completely shed from LC while $\mathrm{N} 2$ is being generated, 375 a direct connection between S3 and N2 can be observed in Figure 9(a). However, at $t U_{\infty} / D=429.7$, this 376 S3-N2 connection breaks up such that a new direct connection is established between S3 and N1 (see 377 Figure 9(c)) due to the faster-moving S-cell vortices in the streamwise direction. Note that the vortex 378 reconnection has not been observed in the wake of a single dual-stepped cylinder, and a possible reason 379 for such an occurrence for the side-by-side, dual stepped cylinders is that the streamwise convection 380 velocity downstream of LC is further reduced by the existence of its neighboring cylinder.

384 X-shaped connection. This has not been observed in the wake of a single dual-stepped cylinder at the 385 same $\mathrm{Re}^{31}$. This difference can be attributed to the higher frequency ratio $f_{S} / f_{N} \approx 2.43-2.76$ in the side386 by-side configuration than $f_{S} / f_{N} \approx 2.26$ in the single-cylinder configuration ${ }^{31}$. When the cylinder [a] 387 has a wider wake, a triple direct connection can even be observed, as shown in Figure 10 with four 388 instants of the repeated triple direct connections. 

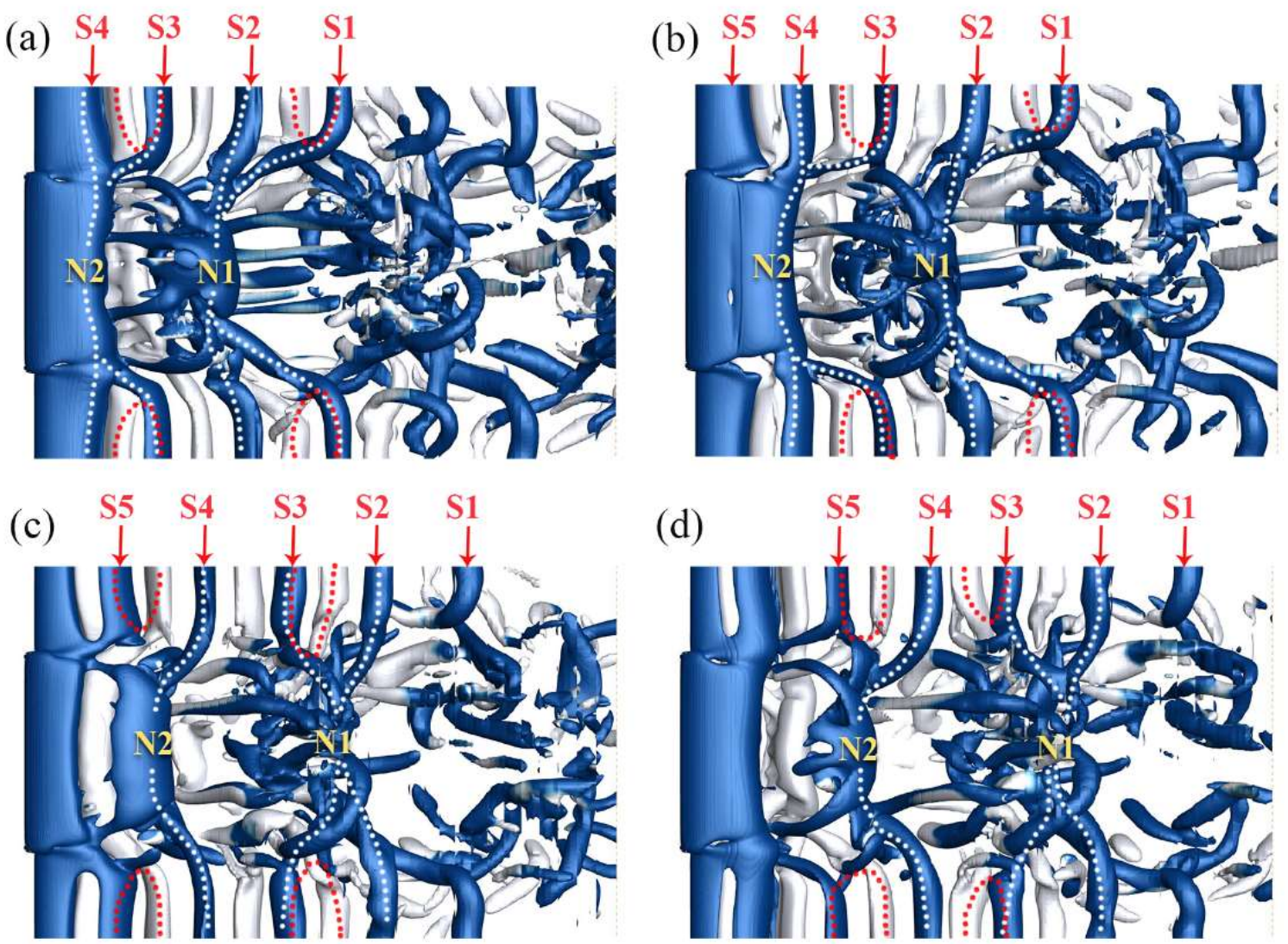

391 Figure 9. Vortex revolution in a narrower wake. Vortices are visalized by the iso-surface of Q=0.2: (a) $392 t U_{\infty} / D=425.7$, (b) $t U_{\infty} / D=427.7$, (c) $t U_{\infty} / D=429.7$, (d) $t U_{\infty} / D=431.7$. The white (red) dotted lines 393 denote the direct (half-loop) connections. S and N denote S-cell and N-cell, respectively, with the 394 sequential numbers of the vortex shedding patterns. 

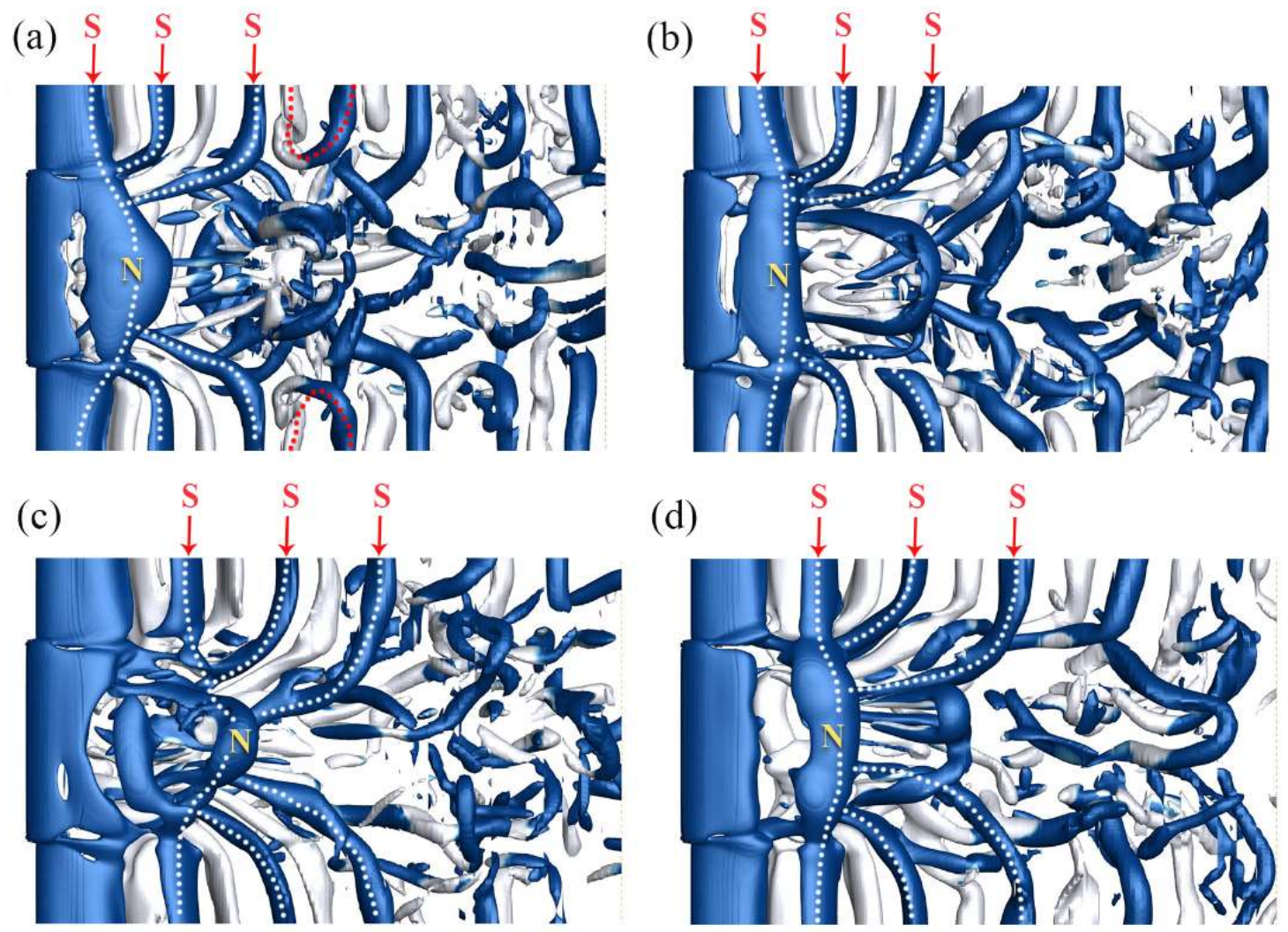

Figure 10. Vortex revolution in a wider wake. Vortices are visalized by the iso-surface of $Q=0.2$ : (a) $t U_{\infty} / D=475.7$, (d) $t U_{\infty} / D=481.7$, (c) $t U_{\infty} / D=715.7$, (d) $t U_{\infty} / D=739.7$. The white (red) dotted lines denote the direct (half-loop) connections. S and N denote S-cell and N-cell, respectively.

Figure 11 shows the simplified drawing of the irregular and aperiodic vortex evolution of cylinder [b] during A3 period associated with the flip-flopping-induced flow instability. It can be reasonably remarked for the narrower wake that the half-loop connection takes place almost at every vortex shedding period: two counter-rotating S-cell vortices (one on the free-stream side and the other from the gap side) are interconnected every two S-cell pairs. This feature appears to be the same as that observed for a single dual-stepped cylinder in Chen et al. ${ }^{45}$. This indicates that the dual-stepped cylinder with the narrower wake behaves like an isolated one. Nevertheless, in the wider wake case, the reconnection and multiple connections frequently appear whereas the half-loop connection is seldomly observed. This difference can be attributed to that, for the wider wake, the transverse distance between two S-cell vortices is relatively larger, enabling them less feasible to connect. Instead, S-cell 
vortices tend to connect L-cell vortices with the same rotating direction to form a multiple direct connection. Note that a similar phenomenon can also be observed in A1 and B1 periods.

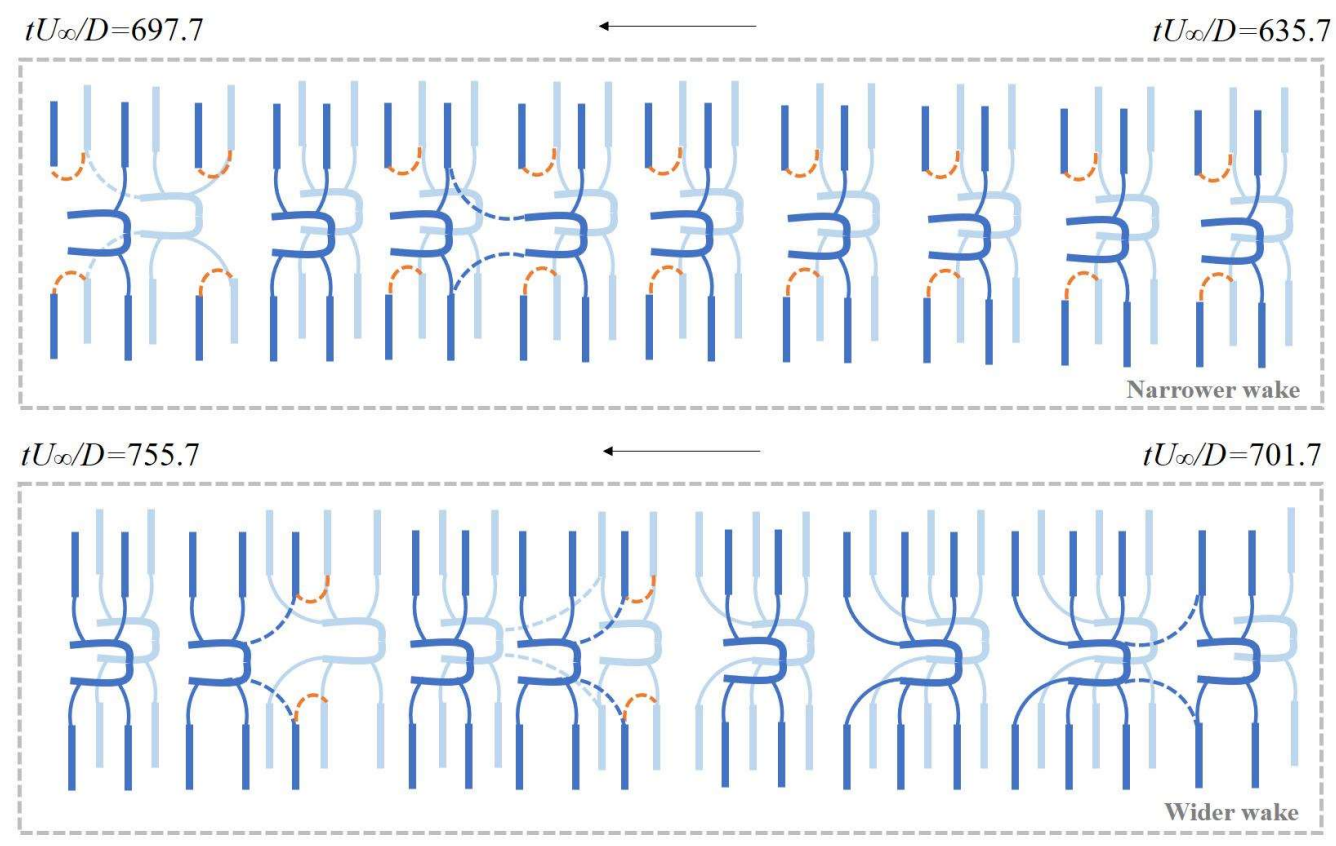

413 Figure 11. Simplified drawings for the vortex connections during A3 ( $\left.t U_{\infty} / D=635.7 \sim 755.7\right)$. Thick 414 lines indicate S-cell and N-cell vortices: straight (mirrored C-shaped) lines denote S-cell (twisted Ncell) vortices. Thin lines represent vortex connections: blue solid, blue dashed and orange dashed lines denote the direct connections, the direct reconnections, and the half-loop connections, respectively. All dark (light) blue lines indicate vortices shed from the free-stream (gap) side.

\subsection{Dynamic mode decomposition}

DMD analyses are carried out for the flip-flopping flow of the two dual-stepped, side-by-side cylinders. Since DMD is sensitive to the collected dataset, the snapshot series are carefully selected in the durations of A3_I $\left(t U_{\infty} / D=636.22 \sim 695.5\right.$ with a gap flow deflecting downward $)$, A3_II $\left(t U_{\infty} / D=\right.$ 706.94 760.5 with a gap flow deflecting upward), and B1 $\left(t U_{\infty} / D=416.22 \sim 509.82\right.$ during which the gap flow switches six times), see Figure 12. The snapshots of non-dimensional z-vorticity (normalized by $U_{\infty} / D$ ) in these periods are extracted with a dimensionless time interval of 0.52 , yielding 181 snapshots for B1, 115 snapshots for A3_I, and 103 snapshots for A3_II. For a two-dimensional analysis, two slices at $z / D=5.5$ and 9.5, downstream of LC and SC, respectively, are selected, on the purpose of investigating the separating flow dynamics. A three-dimensional analysis is then applied to establish 
a connection between the flow dynamics of SC and LC. In addition, spectra of two-dimensional and three-dimensional DMD modes in the three periods are shown in Figure 13. Similar to previous studies $15,16,47$, a cluster of DMD modes with close DMD frequencies do coexist, indicating a complex flow 431 pattern.
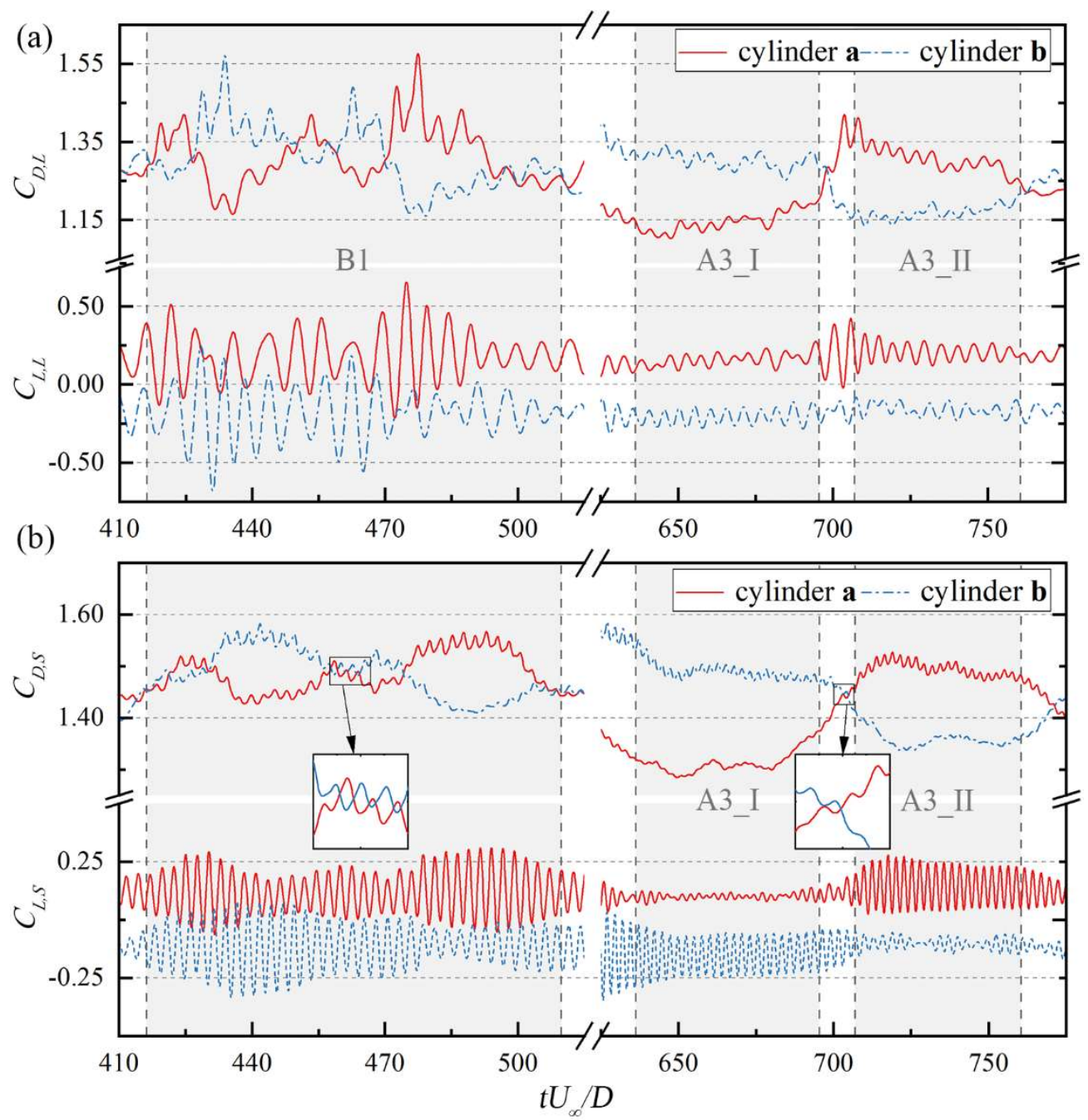

434 Figure 12. Time histories of $C_{D}$ and $C_{L}$ within A3 and B1 durations: (a) LC, (b) SC. 

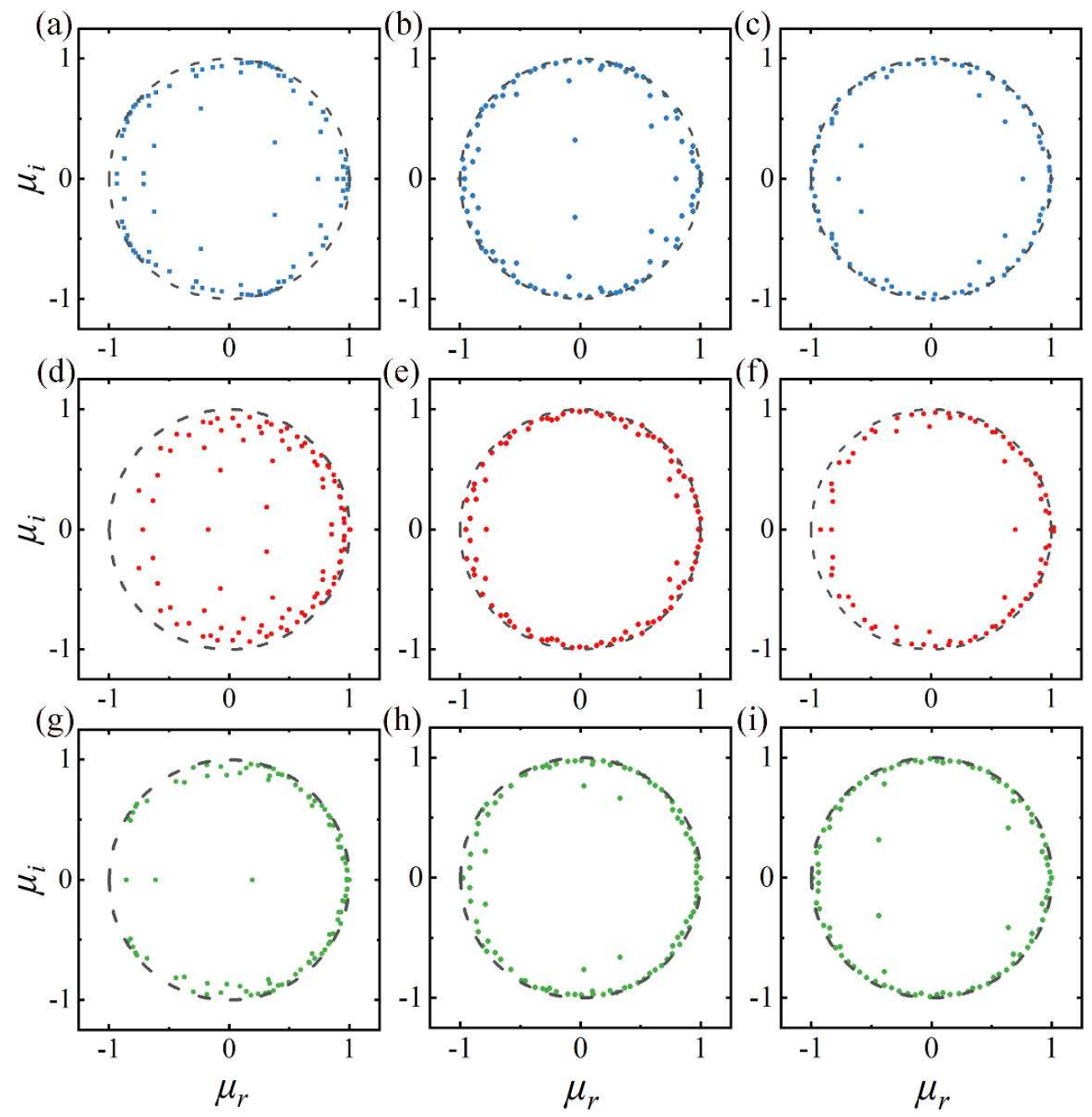

Figure 13. Spectra of two-dimensional DMD modes of SC in (a) B1, (b) A3_I, and (c) A3_II; twodimensional DMD modes of LC in (d) B1, (e) A3_I, and (f) A3_II; three-dimensional DMD modes in (g) B1, (h) A3_I, and (i) A3_II.

Figure 14 shows the mean flow modes of SC and LC for B1 and A3 durations. Some differences are observed. The mean flow mode in B1 manifests a symmetry for both SC and LC, owing to the fact that the gap flow equally defects toward both cylinder sides. However, as the gap flow is consistently biased toward one side in A3_I and A3_II, the mean flow mode has a longer active zone in the wider wake but a shorter one in the narrower wake. This trend becomes more pronunced in the SC wake. 
(a)

(d)
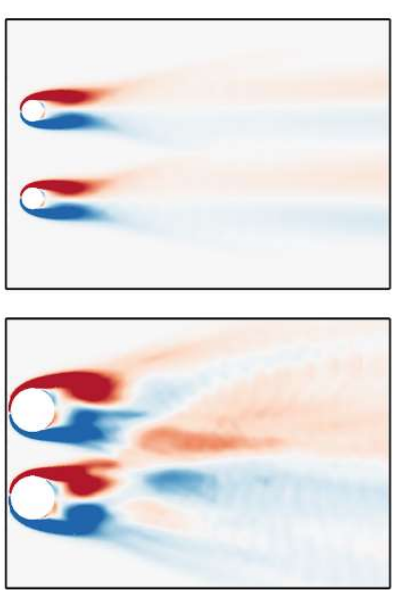

(b)

(e)
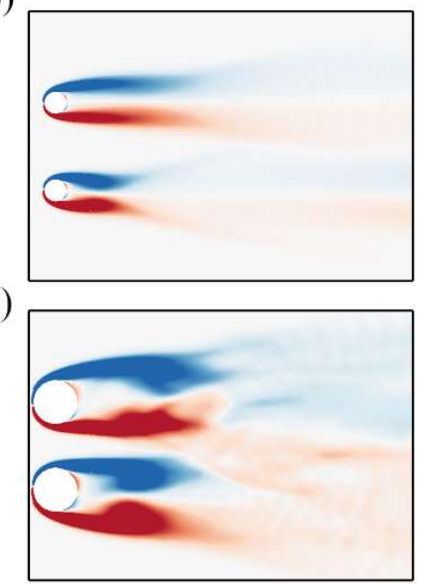

(c)

(f)

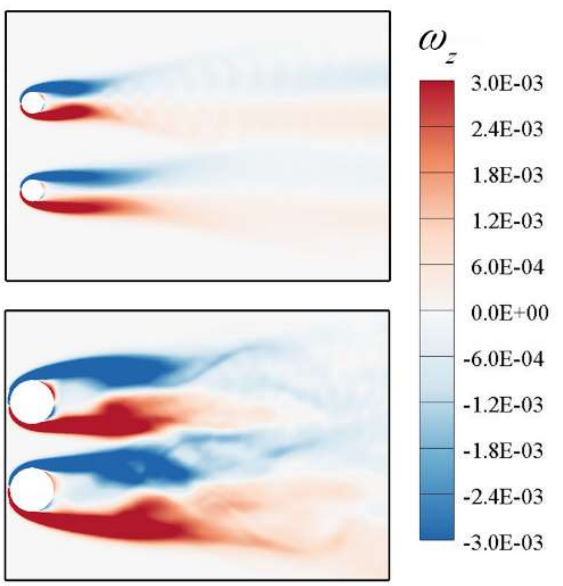

Figure 14. Two-dimensional mean flow modes in A3 and B1 $\left(f_{D M D}=0\right)$ : (a, d) B1, (b, e) A3_I, (c, f) A3_II. The top (bottom) row is associated with SC (LC).

A global mode, which has been reported in previous studies of flow past a circular cylinder ${ }^{34,35}$, also appears in the SC wake, as shown in Figure 15(a-c). In general, this global mode corresponds to the vortex shedding in the wake and, thus, has a frequency equal to the vortex shedding frequency. In this study, as shown in Figure 15(a), the global mode structure, appearing behind both cylinders in B1, has an oscillation frequency $f_{D M D}=0.377$ which is very close to the vortex shedding frequency of SC, $f_{s}=0.370$. However, because the narrower and wider wakes have distinct vortex shedding frequencies, two global modes with asymmetric structures are observed in A3_I. The wider (narrower) wake mode responds with $f_{D M D}=0.310(0.387)$. Note that the global mode at $f_{D M D}=0.310$ only shows a significant disturbance in the wider wake of the upper cylinder (see Figure 15(b)), while the global mode at $f_{D M D}$ $=0.387$ shows the flipped-over structures with disturbances in the narrower wake of the lower cylinder (not shown). The averaged value of DMD frequencies is about 0.349 being close to the vortex shedding frequency $f_{S}=0.356$ of SC in regime A. A similar situation occurs when the gap flow deflects upward, where the corresponding $f_{D M D}=0.330$ for the wider wake of the lower cylinder and $f_{D M D}=0.382$ for the narrower wake of the upper cylinder. Similarly, the second harmonic modes ${ }^{34}$, that oscillate at a frequency twice that of the fundamental vortex shedding, exhibit the same characteristics, as seen in Figure 15(d-f).

The global and second harmonic modes of SC show a similar pattern to the DMD modes in the AS wake of two uniform side-by-side cylinders, except for the asymmetry in A3_I and A3_II during 
which the gap flow is consistently deflected. This implies that the wake pattern downstream of SC is essentially AS, although the hydrodynamic forces of SC and the gap flow show the flip-floppingrelated alterations. These findings are further evidenced by the existence of special DMD modes (see Figure $15(\mathrm{~g}-\mathrm{h}))$ oscillating at $f_{D M D}=0.028(\mathrm{~B} 1), 0.011$ (A3_I) and 0.020 (A3_II) which are very close to the flip-flopping frequencies of 0.054 (regime B) and 0.018 (regime A) in Figure $7(\mathrm{a}, \mathrm{b})$. These intrinsic flip-flopping modes in the absence of an active zone in the gap region are new, being different from the DMD mode related to a pitchfork bifurcation in Liu and Jaiman ${ }^{15,16}$ in which a stationary disturbance exists downstream of the gap. This implies that the flip-flopping behaviors in the SC wake are not self-excited. Note that the flip-flopping-related modes in A3_I and A3_II also exhibit an asymmetry, with the same above-discussed reason.

(a)

(d)
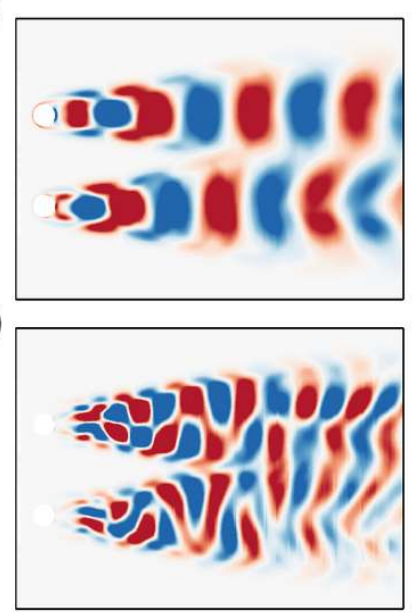

(g)

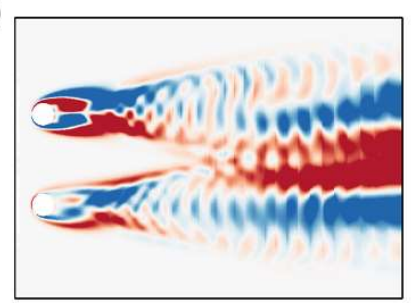

(b)

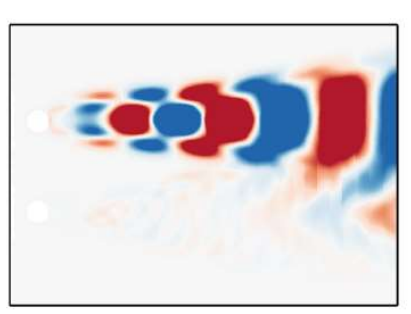

(e)

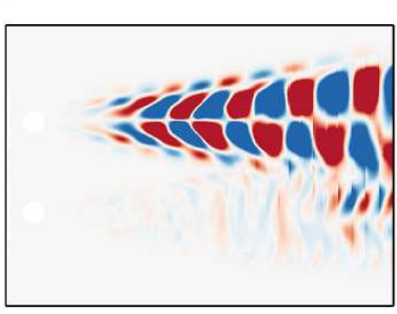

(h)

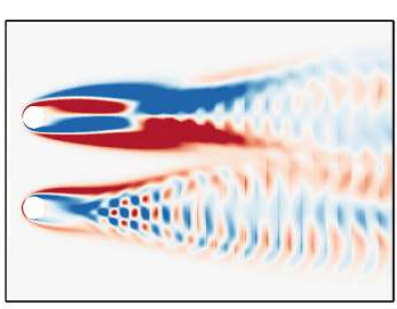

(c)

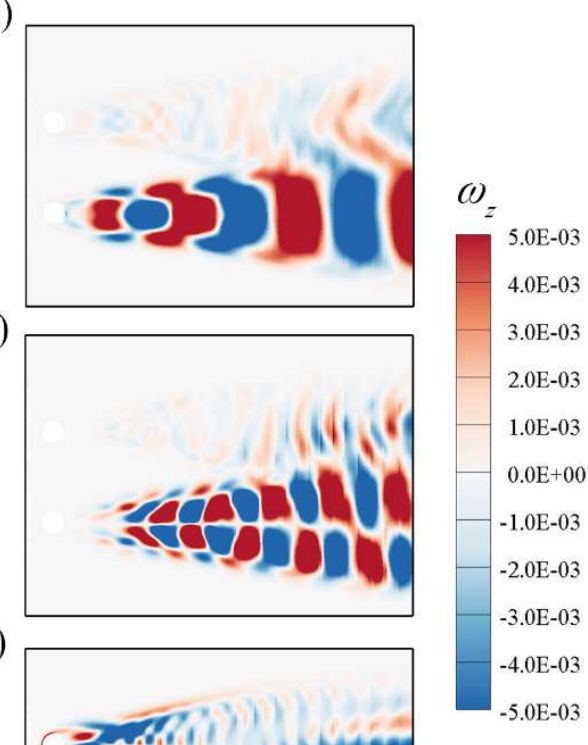

Figure 15. Two-dimensional DMD modes of SC in B1 and A3: global modes in (a) B1 $\left(f_{D M D}=0.377\right)$, (b) A3_I $\left(f_{D M D}=0.310\right)$, (c) A3_II $\left(f_{D M D}=0.330\right)$; second harmonic modes in (d) B1 $\left(f_{D M D}=0.756\right),(\mathrm{e})$ A3_I $\left(f_{D M D}=0.614\right),(\mathrm{f}) \mathrm{A} 3 \_$II $\left(f_{D M D}=0.657\right)$; flip-flopping-related modes in $(\mathrm{g}) \mathrm{B} 1\left(f_{D M D}=0.028\right),(\mathrm{h})$ A3_I $\left(f_{D M D}=0.011\right),(\mathrm{i}) \mathrm{A} 3 \_$II $\left(f_{D M D}=0.020\right)$.

DMD modes in the LC wake are displayed in Figure 16. According to the stability analysis of Mizushima and Ino ${ }^{11}$, a stationary disturbance extending from a gap between side-by-side cylinders toward downstream is a typical feature of the pitchfork bifurcation responsible for a deflected gap flow. 
485 In Figure 16 (a-c), DMD modes reveal some similarities to the pitchfork bifurcation modes presented 486 in Liu and Jaiman ${ }^{16}$. For B1, during which the gap flow switches, the pitchfork bifurcation mode 487 oscillates at a frequency $f_{D M D}=0.018$ being close to $f_{F F}=0.054$. However, in A3_I and A3_II when the 488 gap flow is consistently biased toward one side without switching, this mode either becomes stationary $489\left(f_{D M D}=0\right.$ in A3_I $)$ or oscillates at a rather low frequency $\left(f_{D M D}=0.005\right.$ in A3_II $)$.

490 DMD modes in Figure 16(d-i) show the in-phase (IP) and anti-phase (AP) synchronizations in the 491 near wakes of both cylinders. Both modes are related to the Hopf bifurcations ${ }^{11}$. However, it seems 492 that the AP mode has a higher oscillatiion frequency $\left(f_{D M D}=0.207,0.183,0.176\right.$ in B1, A3_I and A3_II, 493 respectively) than the IP mode $\left(f_{D M D}=0.170,0.129,0.136\right.$ in B1, A3_I and A3_II, respectively $)$. This 494 is consistent with the observation in Chen et al. ${ }^{45}$ where, for a 2-D flow past two side-by-side cylinders, 495 an anti-phase sychronized wake has a higher vortex-shedding frequency than an in-phase sychronized 496 one, owing to the confined space for the development of gap vortices. Although both AP and IP modes show the approximately symmetric active zones behind side-by-side cylinders in B1, IP modes in A3_I and A3_II are predominant in the wider wake with a lower vortex shedding frequency, while AP modes are predominant in the narrower wake with a higher vortex shedding freuquency.

Overall, it is worth remarking that DMD modes corresponding to pitchfork and Hopf bifurcations coexist in the LC wake. As noted in Carini et al. ${ }^{12}$, the flip-flopping flow is originated from an interaction between such two bifurcations. Hence, it is concluded that the flip-flopping behaviors in the LC wake are self-excited. 
(a)

(d)
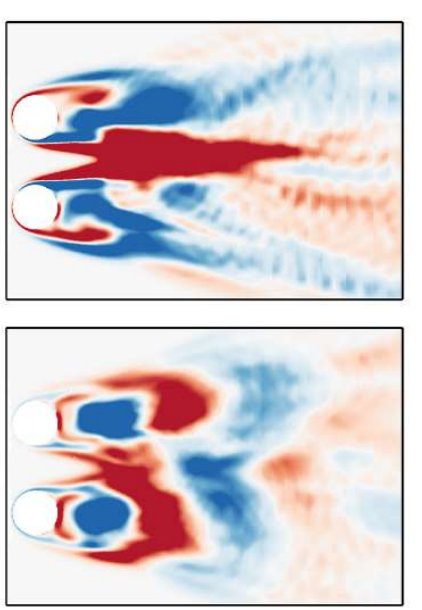

(g)

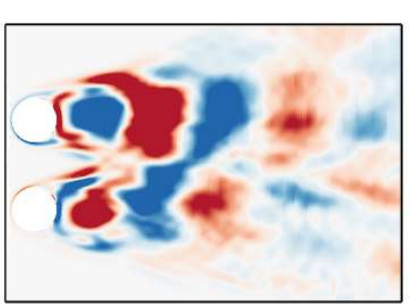

(b)

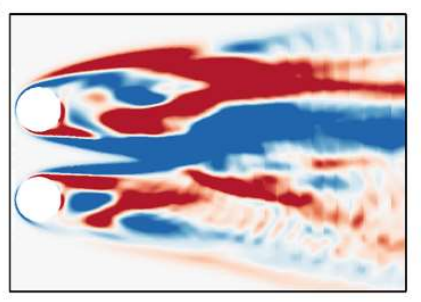

(e)

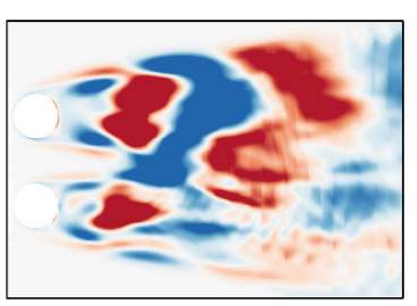

(h)

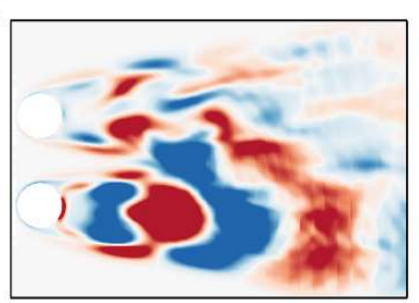

(c)

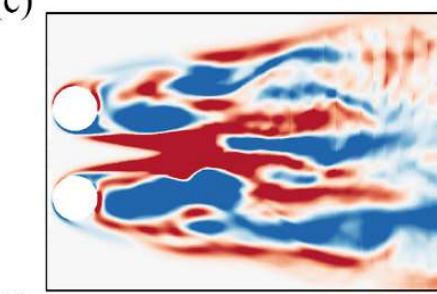

(f)

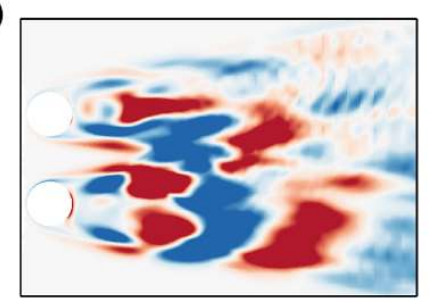

(i)

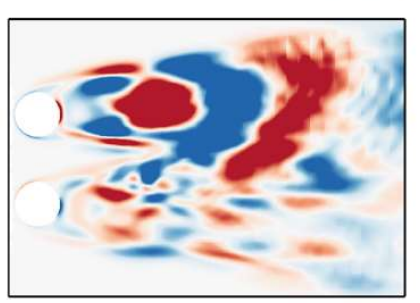

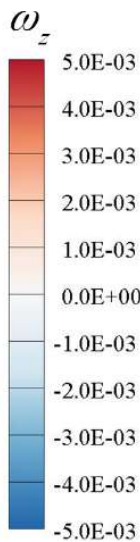

Figure 16. Two-dimensional DMD modes of LC in B1 and A3: pitchfork bifurcation modes in (a) B1 $\left(f_{D M D}=0.018\right),(\mathrm{b}) \mathrm{A} 3 \_\mathrm{I}\left(f_{D M D}=0\right),(\mathrm{c}) \mathrm{A} 3 \_\mathrm{II}\left(f_{D M D}=0.005\right)$; Hopf bifurcation modes (IP) in (d) B1 $\left(f_{D M D}=0.170\right),(\mathrm{e}) \mathrm{A} 3 \_\mathrm{I}\left(f_{D M D}=0.129\right),(\mathrm{f}) \mathrm{A} 3 \_\mathrm{II}\left(f_{D M D}=0.136\right)$; Hopf bifurcation modes $(\mathrm{AP})$ in $(\mathrm{g})$ B1 $\left(f_{D M D}=0.207\right),(\mathrm{h}) \mathrm{A} 3 \_\mathrm{I}\left(f_{D M D}=0.183\right),(\mathrm{i}) \mathrm{A} 3 \_\mathrm{II}\left(f_{D M D}=0.176\right)$.

By comparing DMD modes of LC vs. SC, pitchfork and Hopf bifurcations are only observed in the LC wake. Although SC does show the flip-flopping-related modes, significant differences are observed in the gap region when compared to the pitchfork bifurcation modes of LC. Two DMD modes related to the global and second-order instability are found in the SC wake. However, due to the flipflopping flow-induced modulations, these DMD modes are different from those of two side-by-side uniform cylinders. The underlying physics of the flip-flopping flow of two side-by-side dual-stepped cylinders is that the flip-flopping flow stems from the proximity interference with the spacing ratio of 1.0 for LC; the gap flip-flopping flow behavior is transferred to SC through the vortex connections between N-cell and S-cell. Due to a larger spacing ratio of 3.0 for SC, the intrinsic flow pattern of SC is mainly the AS flow. Nevertheless, because of the effect of the flip-flopping flow induced by LC, the wake pattern of SC also shows a combination of AS (primary) and flip-flopping (secondary) flows. Note that the LC wake pattern can significantly affect the SC wake pattern, but not vice versa. 
The above observations of two-dimensional DMD modes are further supported by the analysis of three-dimensional DMD modes of LC and SC related to the flip-flopping flow. Figure 17(a), 18(a) and 524 19(a) illustrates the mean flow modes sharing similar features to those in Figure 14. Similar to two525 dimensional results, paired DMD modes appear in the SC wake, signifying the existence of Hopf 526 bifurcation, as shown in Figure 17(b), 18(b) and 19(b). Interestingly, in Figure 17(d-f), DMD modes 527 exhibit a direct connection between the pitchfork bifurcation modes of LC and the flip-flopping-related 528 modes of SC. That justifies that the flip-flopping flow appearing in the SC wake is indeed triggered by 529 the pitchfork bifurcation in the LC wake. As exemplified in Figure 17(d), 18(d) and 19(d), the planar modes extracted at $z / D=5.5$ and 9.5 manifest themselves a great similarity to those in Figures $15(\mathrm{~g}-\mathrm{i})$ and $16(a-b)$.

(a)

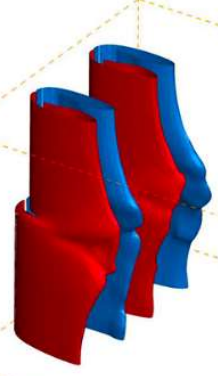

(c)

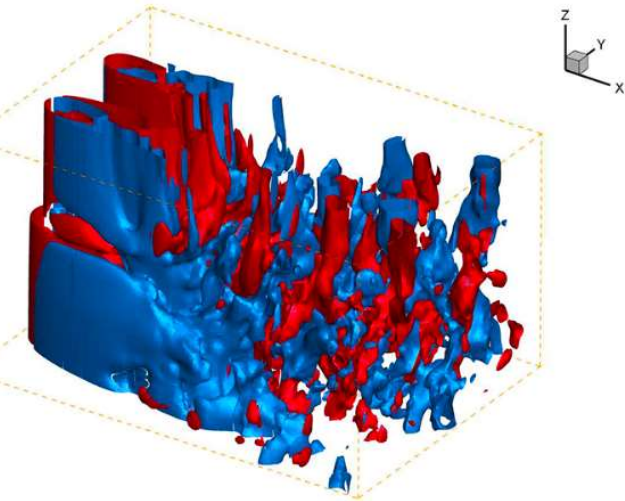

(b)

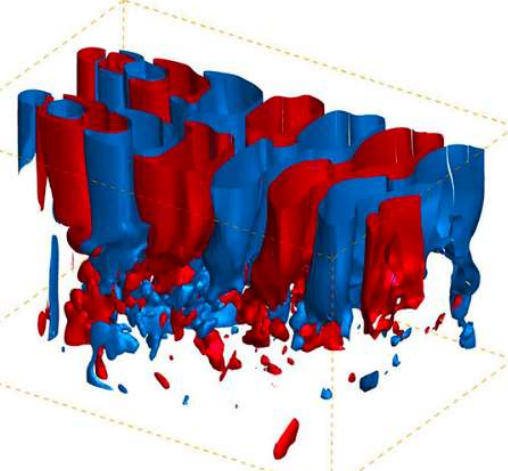

(d)

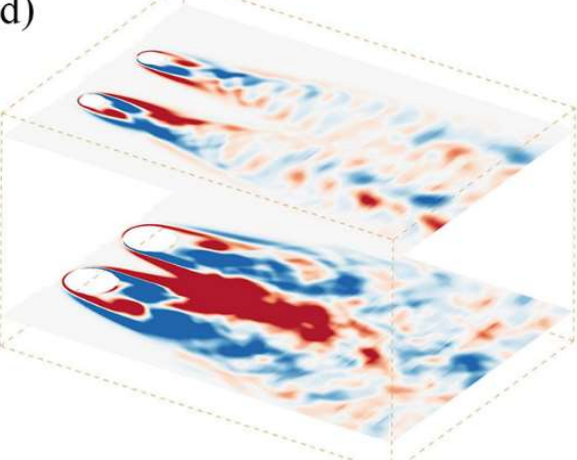

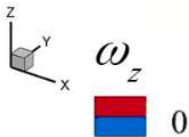

$y^{y}$

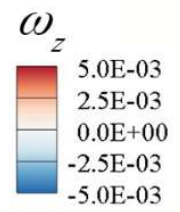

Figure 17. Three-dimensional DMD modes in B1, presented by iso-surfaces of $\omega_{z}=-2.5 \times 10^{-4}$ (blue) and $2.5 \times 10^{-4}$ (red): (a) $f_{D M D}=0$, (b) $f_{D M D}=0.380$, (c) $f_{D M D}=0.0127$, (d) planar modes extracted at $Z / D=5.5$ and $9.5\left(f_{D M D}=0.0127\right)$. 
(a)

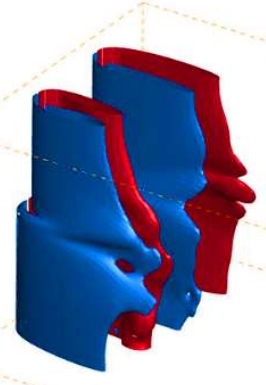

(c)

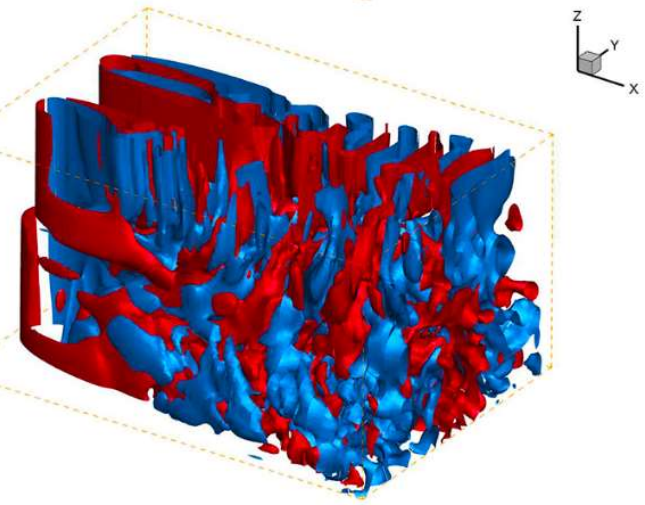

(b)

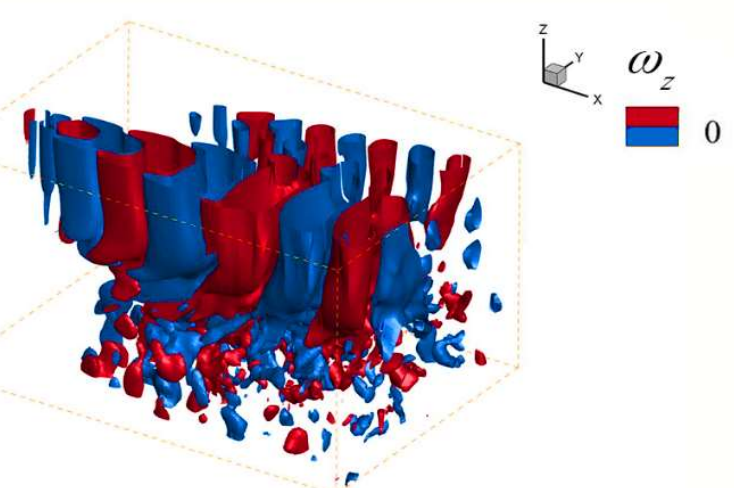

(d)

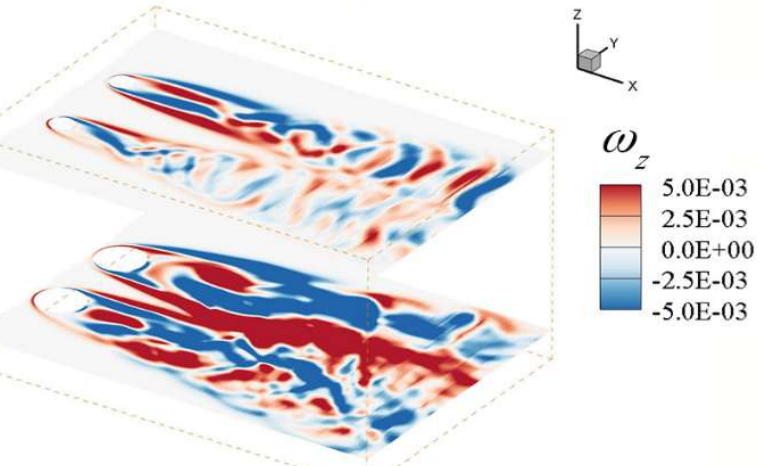

538 Figure 18. Three-dimensional DMD modes in A3_I, presented by iso-surfaces of $\omega_{z}=-2.5 \times 10^{-4}$ (blue) 539 and $2.5 \times 10^{-4}$ (red): (a) $f_{D M D}=0$, (b) $f_{D M D}=0.389$, (c) $f_{D M D}=0.0034$, (d) planar modes extracted at 540 $Z / D=5.5$ and $9.5\left(f_{D M D}=0.0034\right)$. 
(a)

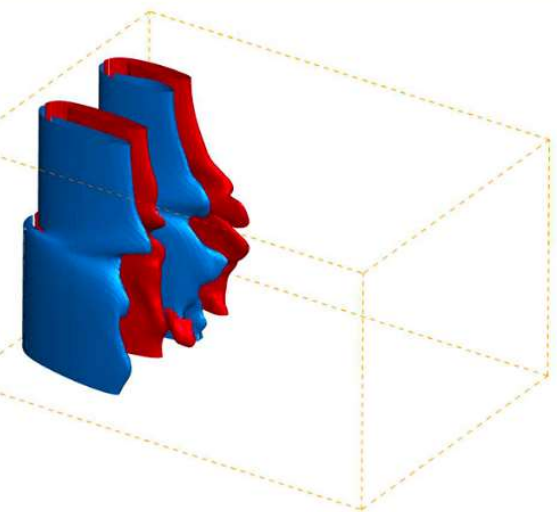

(c)

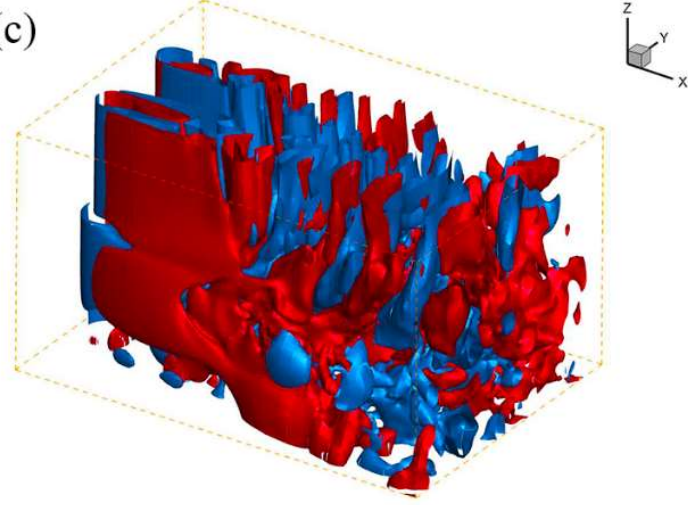

(b)

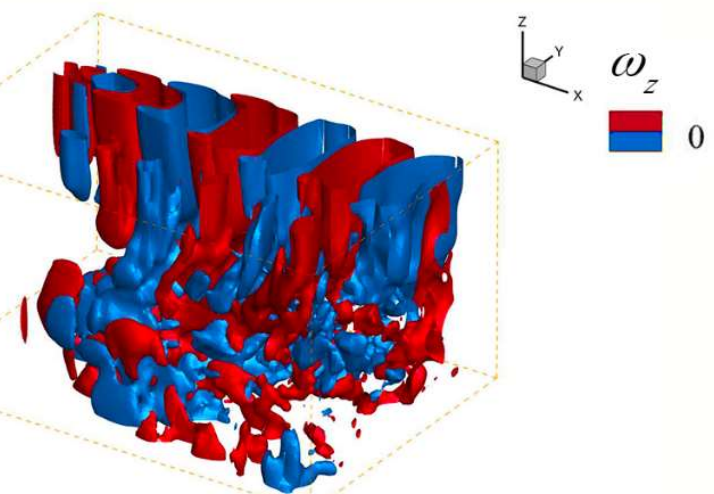

(d)

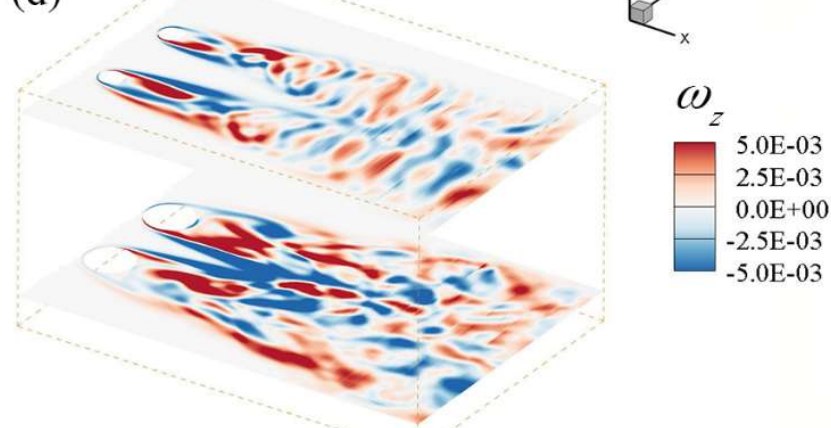

Figure 19. Three-dimensional DMD modes in A3_II, presented by iso-surfaces of $\omega_{z}=-2.5 \times 10^{-4}$ (blue) and $2.5 \times 10^{-4}$ (red): (a) $f_{D M D}=0$, (b) $f_{D M D}=0.387$, (c) $f_{D M D}=0.0105$, (d) planar modes extracted at $Z / D=5.5$ and $9.5\left(f_{D M D}=0.0105\right)$.

\section{Conclusions}

Three-dimensional direct numerical simulations and the dynamic mode decomposition analyses have been carried out to investigate the wake patterns and stability of a pair of dual-stepped circular cylinders in a side-by-side arrangement and a proximity interference region. The dual-stepped cylinders consist of two coaxial cylinders with a larger-to-smaller diameter ratio of 2 . The Reynolds number and the side-by-side gap ratio, based on the larger (smaller) diameter, is 200 (100) and 1 (3), respectively. The middle cylinders with larger diameters exhibit the flip-flipping flow behaviors. The top and bottom cylinders with smaller diameters reveal a combination of anti-phase synchronized (primary) and flip-flopping (secondary) flows. The intrinsic features of associated wake patterns have been discussed, highlighting the underlying physics of three-dimensional flow around the side-by-side nonuniform cylinders in comparison with those of the side-by-side uniform cylinders in the literature. 
Two distinct flow regimes (A and B) appear in sequence for both smaller and larger cylinders. The 558 flow regime B entails greater drag and lift force coefficients as well as greater flip-flopping and vortex 559 shedding frequencies. A clear separation between different flow stages can be observed in regime A, 560 rather than in regime B which is subject to a faster and irregular switching of the gap flow. Greater 561 phase fluctuations and jumps of drag and lift coefficients frequently take place in the flow regime B. 562 Three-dimensional vortex structures in the wider versus narrower wake are different. In addition to the 563 direct and half-loop connections of vortices, two new vortex connections have been identified as the vortex reconnection and multiple connection, which are predominant in the wider wake regime. Both the half-loop and direct connections mostly coexist in the narrower wake, indicating that the wake structures in the narrower wake are analogous to those of a single dual-stepped cylinder.

Two-dimensional dynamic mode decomposition (DMD) modes have been characterized. In the wake of the larger cylinder, DMD modes related to the deflected gap flow are associated with the pitchfork bifurcations whereas the modes related to in-phase and anti-phase synchronizations are associated with the Hopf bifurcations. Nevertheless, none of these DMD modes is found in the wake of the smaller cylinder, which reveals global and second harmonic modes as in the case of two sideby-side uniform cylinders. However, due to the flip-flopping flow-induced modulations, these DMD modes of nonuniform cylinders are different from those of uniform cylinders. The underlying physics of the flip-flopping flow around a pair of side-by-side, dual-stepped cylinders is that the flip-flopping 575 flow stems from the proximity interference between the two middle cylinders with larger diameters; then, the gap flip-flopping flow mechanism is transferred through the vortex connections between $\mathrm{N}$ cell and S-cell to the top and bottom cylinders with smaller diameters. Due to a larger spacing ratio and the influence of flip-flopping flow induced by larger cylinders, the wake patterns of smaller cylinders exhibit a combination of anti-phase synchronization (primary) and flip-flopping (secondary) flows. Interestingly, a three-dimensional DMD analysis captures a direct connection between the pitchfork bifurcation mode of the larger cylinder and the flip-flopping mode of the smaller cylinder. 


\section{Appendix}

584

585

Supplementary results of flow past a pair of side-by-side dual-stepped cylinders with $D / d=1.33$ are presented. In this case, we keep the diameter of SC unchanged but reduce the diameter of LC, resulting in a smaller $\operatorname{Re}_{D}=133.3$ and a larger $g^{*}{ }_{D}=2$. For $\mathrm{SC}, \operatorname{Re}_{d}=100$ and $g^{*}{ }_{d}=3$, being identical to those in Section 3. According to the flow pattern partition of two side-by-side uniform cylinders in Kang ${ }^{10}$, both two configurations trigger the anti-phase synchronization (AS) pattern which is stable and periodic. As shown in Figure A1(f), in spite of the vortex distortions caused by the different streamwise velocity of S-cell and N-cell, the vortex shedding of LC and SC is in the anti-phase synchronized pattern, with the dominant direct connections. The vortex dislocations are only observed in the far downstream, signifying the weak interactions of S-cell and N-cell vortices. As stated in McClure et al. ${ }^{48}$, in a single dual-stepped cylinder case, the increased diameter ratio can make the dislocation between $\mathrm{N}-c e l l$ and S-cell become less pronounced. This is consistent with the present observation.

Figures A1(a-d) further illustrate time histories of force coefficients for $t U_{o} / D=128 \sim 268$. It is seen that the force responses show stable and periodic variations in time. These are clearly different from the irregular and aperiodic force time histories in the FF regime. However, there exists significant low-frequency force components, manifesting the beating characteristics of force coefficients. According to the PSD spectrum (probed at $x / D=5, y / D=2$ ) in Figure A1(e), the beating frequency is actually the frequency of dislocation $\left(f_{\text {dis }}\right)$ between $\mathrm{N}$-cell and S-cell, i.e. $f_{\text {dis }}=f_{S-} f_{N}=0.057$ with $f_{N}=$ 0.184 and $f_{S}=0.241$. 

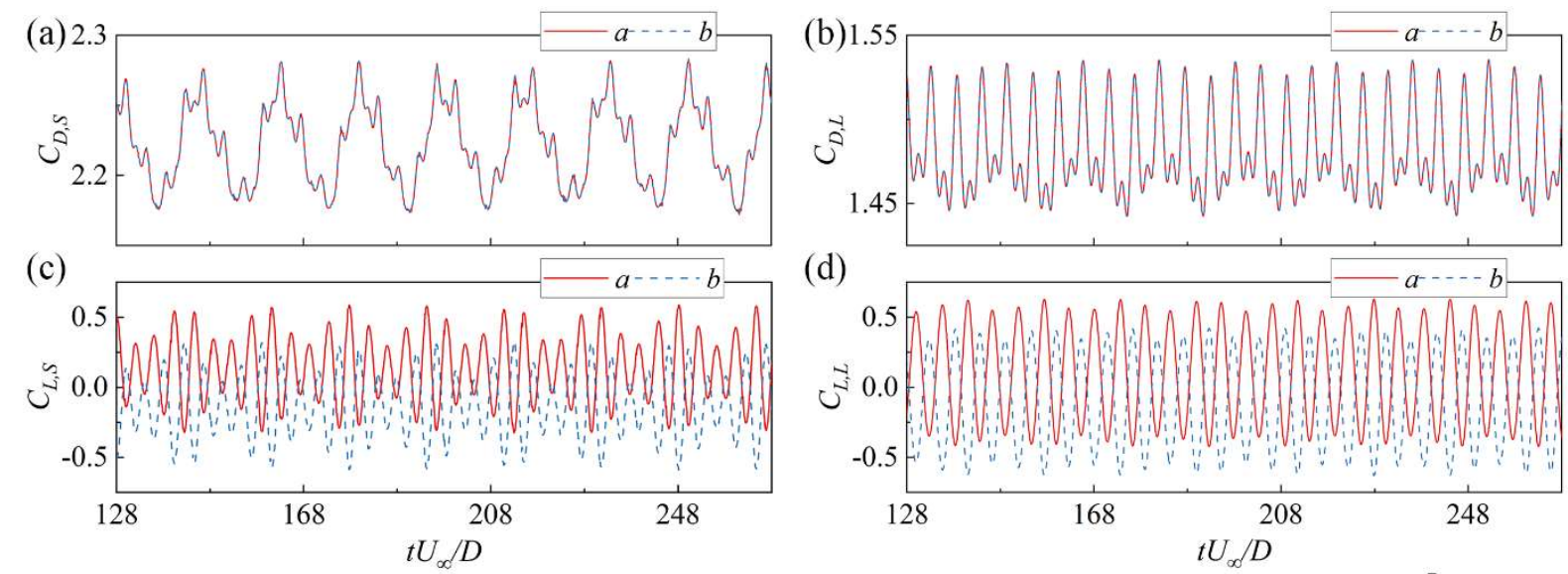

(d)

(e)

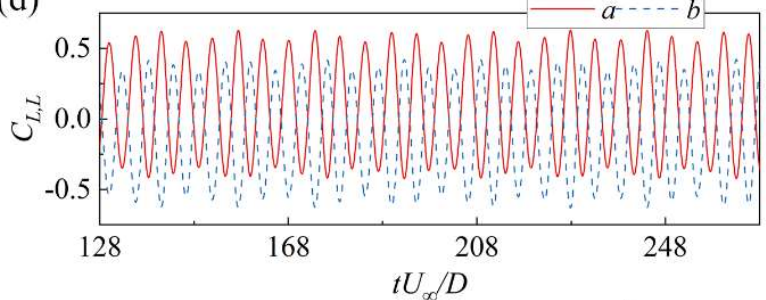

(f)
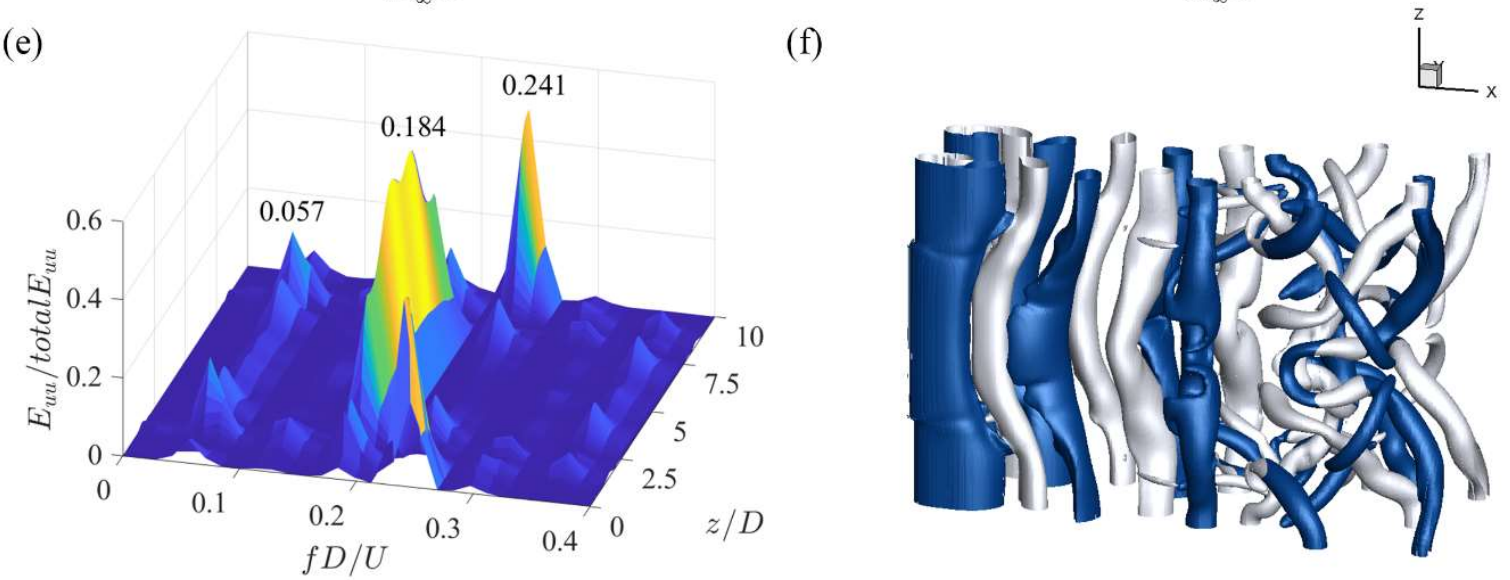

603 Figure A1. Flow past two side-by-side dual-stepped cylinders with $D / d=1.33$. Drag coefficient: (a) 604 smaller and (b) larger cylinders; lift coefficient: (c) smaller and (d) larger cylinders; (e) PSD spectrum 605 of the normalized energy density $E_{u u}$ in the streamwise direction during $t U_{\infty} / D=128 \sim 268$; (f) 606 instantaneous vortex structures, visualized by the iso-surface of the $\mathrm{Q}$-criterion at $\mathrm{Q}=0.2$.

\section{Acknowledgments}

This work was financially supported by the National Natural Science Foundation of China (Grant 609 No. 51779172) and the National Key Research and Development Program of China (Grant No. 2017YFC1404200). The work was carried out at the National Supercomputer Center in Tianjin, and 611 the calculations were performed in Tianhe 3 prototype. 
The data that support the findings of this study are available from the corresponding author upon reasonable request.

\section{REFERENCES}

1. M. M. Zdravkovich, "The effects of interference between circular cylinders in cross flow," Journal of Fluids and Structures 1 (2), 239-261 (1987).

618 2. D. Sumner, "Two circular cylinders in cross-flow: A review," Journal of Fluids and Structures 26 619 (6), 849-899 (2010).

620 3. J. C. Lin, Y. Yang and D. Rockwell, "Flow past two cylinders in tandem: instantaneous and 621 averaged flow structure," Journal of Fluids and Structures 16 (8), 1059-1071 (2002).

622 4. D. Sumner, S. S. T. Wong, S. J. Price and M. P. PaÏDoussis, "Fluid behaviour of side-by-side 623 circular cylinders in steady cross-flow," Journal of Fluids and Structures 13 (3), 309-338 (1999).

624 5. S. Ishigai, E. Nishikawa, K. Nishimura and K. Cho, "Experimental Study on Structure of Gas Flow 625 in Tube Banks with Tube Axes Normal to Flow : Part 1, Karman Vortex Flow from Two Tubes at 626 Various Spacings," Bulletin of JSME 15 (86), 949-956 (1972).

627 6. P. W. Bearman and A. J. Wadcock, "The interaction between a pair of circular cylinders normal to 628 a stream," Journal of Fluid Mechanics 61 (3), 499-511 (1973).

629 7. C. H. K. Williamson, "Evolution of a single wake behind a pair of bluff bodies," Journal of Fluid 630 Mechanics 159, 1-18 (1985).

631 8. Y. Zhou, H. J. Zhang and M. W. Yiu, "The turbulent wake of two side-by-side circular cylinders," 632 Journal of Fluid Mechanics 458, 303-332 (2002).

633 9. H. J. Kim and P. A. Durbin, "Investigation of the flow between a pair of circular cylinders in the 634 flopping regime," Journal of Fluid Mechanics 196, $431-448$ (1988).

635 10. S. Kang, "Characteristics of flow over two circular cylinders in a side-by-side arrangement at low 636 Reynolds numbers," Physics of Fluids 15 (9), 2486-2498 (2003).

637 11. J. Mizushima and Y. Ino, "Stability of flows past a pair of circular cylinders in a side-by-side 638 arrangement," Journal of Fluid Mechanics 595, 491-507 (2008).

639 12. M. Carini, F. Giannetti and F. Auteri, "On the origin of the flip-flop instability of two side-by-side 640 cylinder wakes," Journal of Fluid Mechanics 742, 552-576 (2014).

641 13. C. W. Rowley, I. MeziĆ, S. Bagheri, P. Schlatter and D. S. Henningson, "Spectral analysis of 642 nonlinear flows," Journal of Fluid Mechanics 641, 115-127 (2009).

643 14. P. J. Schmid, "Dynamic mode decomposition of numerical and experimental data," Journal of 644 Fluid Mechanics 656, 5-28 (2010). 
15. B. Liu and R. K. Jaiman, "Dynamics and stability of gap-flow interference in a vibrating side-byside arrangement of two circular cylinders," Journal of Fluid Mechanics 855, 804-838 (2018).

16. B. Liu and R. K. Jaiman, "Interaction dynamics of gap flow with vortex-induced vibration in sideby-side cylinder arrangement," Physics of Fluids 28 (12), 127103 (2016).

17. K. Lam, F. H. Wang and R. M. C. So, "Three-dimensional nature of vortices in the near wake of a wavy cylinder," Journal of Fluids and Structures 19 (6), 815-833 (2004).

18. H. Nakamura and T. Igarashi, "Omnidirectional reductions in drag and fluctuating forces for a circular cylinder by attaching rings," Journal of Wind Engineering and Industrial Aerodynamics 96 (6), 887-899 (2008).

19. C. H. K. Williamson, "Oblique and parallel modes of vortex shedding in the wake of a circular cylinder at low Reynolds numbers," Journal of Fluid Mechanics 206, 579-627 (1989).

20. W. Dunn and S. Tavoularis, "Experimental studies of vortices shed from cylinders with a stepchange in diameter," Journal of Fluid Mechanics 555, 409-437 (2006).

21. C. G. Lewis and M. Gharib, "An exploration of the wake three dimensionalities caused by a local discontinuity in cylinder diameter," Physics of Fluids A: Fluid Dynamics 4 (1), 104-117 (1992).

22. C. Morton and S. Yarusevych, "Vortex Dynamics in the Turbulent Wake of a Single Step Cylinder," Journal of Fluids Engineering 136 (3) (2014).

23. B. Vallès, H. I. Andersson and C. B. Jenssen, "Direct-mode interactions in the wake behind a stepped cylinder," Physics of Fluids 14 (4), 1548-1551 (2002).

24. C. Morton and S. Yarusevych, "Vortex shedding in the wake of a step cylinder," Physics of Fluids 22 (8), 083602 (2010).

25. T. Cai, F. Jiang, B. Pettersen and H. Andersson, "Antisymmetric vortex interactions in the wake behind a step cylinder," Physics of Fluids 29, 101704 (2017).

26. C. Tian, F. Jiang, B. Pettersen and H. I. Andersson, "Vortex dislocation mechanisms in the near wake of a step cylinder," Journal of Fluid Mechanics 891 (2020).

27. C. H. K. Williamson, "The natural and forced formation of spot-like 'vortex dislocations' in the transition of a wake," Journal of Fluid Mechanics 243, 393-441 (1992).

28. C. Morton and S. Yarusevych, "An experimental investigation of flow past a dual step cylinder," Experiments in Fluids 52, 69-83 (2012).

29. C. Morton, S. Yarusevych and F. Scarano, "A tomographic particle image velocimetry investigation of the flow development over dual step cylinders," Physics of Fluids 28, 025104 (2016). 30. C. Morton and S. Yarusevych, "Vortex shedding from cylinders with two step discontinuities in diameter," Journal of Fluid Mechanics 902, A29 (2020).

31. C. Ji, X. Yang, Y. Yu, Y. Cui and N. Srinil, "Numerical simulations of flows around a dual step cylinder with different diameter ratios at low Reynolds number," European Journal of Mechanics B/Fluids 79, 332-344 (2020).

32. C. Ji, Y. Cui, D. Xu, X. Yang and N. Srinil, "Vortex-induced vibrations of dual-step cylinders with different diameter ratios in laminar flows," Physics of Fluids 31 (7), 073602 (2019). 

Computer Physics Communications 192, 205-219 (2015).

34. H. Xu, C. D. Cantwell, C. Monteserin, C. Eskilsson, A. P. Engsig-Karup and S. J. Sherwin, "Spectral/hp element methods: Recent developments, applications, and perspectives," Journal of Hydrodynamics 30 (1), 1-22 (2018).

35. D. Moxey, C. D. Cantwell, Y. Bao, A. Cassinelli, G. Castiglioni, S. Chun, E. Juda, E. Kazemi, K. Lackhove, J. Marcon, G. Mengaldo, D. Serson, M. Turner, H. Xu, J. Peiró, R. M. Kirby and S. J. Sherwin, "Nektar++: Enhancing the capability and application of high-fidelity spectral/hp element methods," Computer Physics Communications 249, 107110 (2020).

36. G. E. Karniadakis and S. J. Sherwin, Spectral/Hp Element Methods for Computational Fluid Dynamics, Oxford University Press, 2005.

37. G. E. Karniadakis, M. Israeli and S. A. Orszag, "High-order splitting methods for the incompressible Navier-Stokes equations," Journal of Computational Physics 97 (2), 414-443 (1991). 38. J. L. Guermond and J. Shen, "Velocity-Correction Projection Methods for Incompressible Flows," SIAM Journal on Numerical Analysis 41 (1), 112-134 (2003).

39. J. N. Kutz, S. L. Brunton, B. W. Brunton and J. L. Proctor, Dynamic Mode Decomposition, Society for Industrial and Applied Mathematics, 2016.

40. X. Wang, R. M. C. So and W.-C. Xie, "Wavelet Analysis of Flow-Induced Forces on Two Sideby-Side Stationary Cylinders: Reynolds Number Effect", in Procedings of the the ASME Pressure Vessels and Piping/ICPVT-11 Conference, Vancouver, BC, Canada, 23-27, 2006.

41. D. S. Lee, M. Y. Ha, H. S. Yoon and S. Balachandar, "A numerical study on the flow patterns of two oscillating cylinders," Journal of Fluids and Structures 25 (2), 263-283 (2009).

42. W. Chen, C. Ji, R. Wang, D. Xu and J. Campbell, "Flow-induced vibrations of two side-by-side circular cylinders: Asymmetric vibration, symmetry hysteresis and near-wake patterns," Ocean Engineering 110, 244-257 (2015).

43. W. Chen, C. Ji and D. Xu, "Vortex-induced vibrations of two side-by-side circular cylinders with two degrees of freedom in laminar cross-flow," Computers \& Fluids 193, 104288 (2019).

44. W. Chen, C. Ji, D. Xu and N. Srinil, "Wake patterns of freely vibrating side-by-side circular cylinders in laminar flows," Journal of Fluids and Structures 89, 82-95 (2019). 45. W. Chen, C. Ji, M. M. Alam, J. Williams and D. Xu, "Numerical simulations of flow past three circular cylinders in equilateral-triangular arrangements," Journal of Fluid Mechanics 891, A14 (2020). 46. S. L. Miller and D. Childers, in Probability and Random Processes, edited by S. L. Miller and D. Childers (Academic Press, Burlington, 2004), pp. 369-411. 726, 596-623 (2013).

48. J. McClure, C. Morton and S. Yarusevych, "Flow development and structural loading on dual step 
721 cylinders in laminar shedding regime," Physics of Fluids 27 (6), 063602 (2015). 